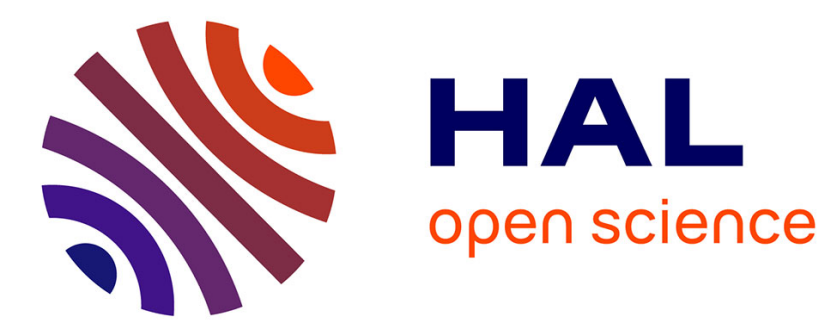

\title{
Linear biglobal analysis of Rayleigh-Bénard instabilities in binary fluids with and without throughflow
}

\author{
Jun Hu, Daniel Henry, Xie-Yuan Yin, Hamda Ben Hadid
}

\section{To cite this version:}

Jun Hu, Daniel Henry, Xie-Yuan Yin, Hamda Ben Hadid. Linear biglobal analysis of Rayleigh-Bénard instabilities in binary fluids with and without throughflow. Journal of Fluid Mechanics, 2012, 713, pp.216-242. 10.1017/jfm.2012.455 . hal-00780152

\section{HAL Id: hal-00780152 \\ https://hal.science/hal-00780152}

Submitted on 2 Oct 2013

HAL is a multi-disciplinary open access archive for the deposit and dissemination of scientific research documents, whether they are published or not. The documents may come from teaching and research institutions in France or abroad, or from public or private research centers.
L'archive ouverte pluridisciplinaire HAL, est destinée au dépôt et à la diffusion de documents scientifiques de niveau recherche, publiés ou non, émanant des établissements d'enseignement et de recherche français ou étrangers, des laboratoires publics ou privés. 


\title{
Linear biglobal analysis of Rayleigh-Bénard instabilities in binary fluids with and without throughflow
}

\author{
Jun $\mathrm{Hu}^{1} \dagger$, Daniel Henry ${ }^{2}$, Xie-Yuan Yin $^{3}$ and Hamda BenHadid ${ }^{2}$ \\ ${ }^{1}$ Institute of Applied Physics and Computational Mathematics, Beijing 100088, China \\ ${ }^{2}$ Laboratoire de Mécanique des Fluides et d'Acoustique, CNRS/Université de Lyon, Ecole Centrale de \\ Lyon/Université Lyon 1/INSA de Lyon, ECL, 36 avenue Guy de Collongue, 69134 Ecully CEDEX, \\ France \\ ${ }^{3}$ Department of Modern Mechanics, University of Science and Technology of China, Hefei 230027,
} China

(Received 4 January 2012; revised 31 July 2012; accepted 14 September 2012; first published online 19 October 2012)

Three-dimensional Rayleigh-Bénard instabilities in binary fluids with Soret effect are studied by linear biglobal stability analysis. The fluid is confined transversally in a duct and a longitudinal throughflow may exist or not. A negative separation factor $\psi=-0.01$, giving rise to oscillatory transitions, has been considered. The numerical dispersion relation associated with this stability problem is obtained with a twodimensional Chebyshev collocation method. Symmetry considerations are used in the analysis of the results, which allow the classification of the perturbation modes as $S_{l}$ modes (those which keep the left-right symmetry) or $R_{x}$ modes (those which keep the symmetry of rotation of $\pi$ about the longitudinal mid-axis). Without throughflow, four dominant pairs of travelling transverse modes with finite wavenumbers $k$ have been found. Each pair corresponds to two symmetry degenerate left and right travelling modes which have the same critical Rayleigh number $R a_{c}$. With the increase of the duct aspect ratio $A$, the critical Rayleigh numbers for these four pairs of modes decrease and closely approach the critical value $R a_{c}=1743.894$ obtained in a twodimensional situation, one of the mode (a $S_{l}$ mode called mode A) always remaining the dominant mode. Oscillatory longitudinal instabilities $(k \approx 0)$ corresponding to either $S_{l}$ or $R_{x}$ modes have also been found. Their critical curves, globally decreasing, present oscillatory variations when the duct aspect ratio $A$ is increased, associated with an increasing number of longitudinal rolls. When a throughflow is applied, the symmetry degeneracy of the pairs of travelling transverse modes is broken, giving distinct upstream and downstream modes. For small and moderate aspect ratios $A$, the overall critical Rayleigh number in the small Reynolds number range studied is only determined by the upstream transverse mode A. In contrast, for larger aspect ratios as $A=7$, different modes are successively dominant as the Reynolds number is increased, involving both upstream and downstream transverse modes A and even the longitudinal mode.

Key words: buoyancy-driven instability, double diffusive convection 


\section{Introduction}

Laminar forced and mixed convection of binary fluids in a horizontal duct heated from below has many practical technological applications such as the cooling process of electronic devices and the techniques of chemical vapour deposition (CVD) for the production of magnetic and optic data storage devices in the electronics industry. It also leads to a variety of spatiotemporal patterns, the study of which has great theoretical interest. In fact, the spatiotemporal behaviour of the dissipative structures appearing in binary mixture convection (Cross \& Hohenberg 1993) has been revealed to be complex due to the combination of thermal forcing (characterized by the Rayleigh number $R a$ ) and Soret coupling between temperature and concentration fields (characterized by the separation factor $\psi$ ); and the externally imposed throughflow (characterized by the Reynolds number $R e$ ) will further break the symmetries existing in the pure Rayleigh-Bénard case.

Jung, Lücke \& Büchel (1996) were the first to investigate how a horizontal plane Poiseuille shear flow changes linear convection properties in binary fluid layers heated from below. They solved the full linear stability equations by a shooting method for realistic top and bottom boundary conditions. For negative Soret coupling, they elucidated the throughflow lifting of the Hopf symmetry degeneracy of left and right travelling waves (TWs). They also showed how the frequencies, bifurcation thresholds and structural properties of the two TW solution branches, as well as the stationary overturning convection (SOC), were dramatically changed when a throughflow was applied. Later, Büchel \& Lücke (2000a) investigated the effect of a horizontal Poiseuille throughflow on stationary and TW convective patterns that appear for negative Soret coupling. The numerical calculations in a two-dimensional periodic box containing two counter-rotating near-critical transverse rolls are performed with a Galerkin expansion or a finite-difference numerical method. Bifurcation diagrams of various quantities such as the Nusselt number, frequency and mixing behaviour are determined as functions of heating rate and wavenumber for several throughflow rates and Soret coupling strengths for ethanol-water parameters. They also studied the growth dynamics of small convective perturbations into different, strongly nonlinear convective states and the transition between them. Concerning the characterization of the absolute or convective nature of the instabilities in such flows, the boundary curves separating these two types of instabilities for both negative separation factors (corresponding to the two TW solutions) and positive separation factors (corresponding to the SOC solution) are first plotted as a function of the throughflow rate in the paper of Jung et al. (1996). Büchel \& Lücke (2000b) then studied the linear spatiotemporal properties of spatially localized convective perturbations for heated binary fluid layers, with or without throughflow. Fronts and pulse-like wave packets formed out of the three relevant perturbations (two oscillatory ones and a stationary one) are analysed after evaluating the appropriate saddle points of the three respective dispersion relations of the linear stability equations over the complex wavenumber plane. Some comparisons with pulses and fronts obtained by numerical simulation are also given. Finally, Jung \& Lücke (2005) investigated the nonlinear evolution of TW fronts and localized TW convection in binary fluid mixtures with strongly negative Soret coupling. They used numerical simulations performed in two-dimensional longextent cavities to point out and elucidate the differences in the evolutions of fronts and localized waves. By using the Chebyshev collocation method to solve the full linear stability equations, Hu, Ben Hadid \& Henry (2007) extended the study of the temporal and spatiotemporal instabilities in the Poiseuille-Rayleigh-Bénard flow with Soret effect to larger Reynolds numbers. They considered both positive and negative 
separation factors in the usual situation when heating is from below, but also negative separation factors when heating is from the top. They pointed out parameter ranges where transitions between different critical wave patterns may occur. Thanks to twodimensional numerical simulations initiated by pulse-like disturbances, $\mathrm{Hu}$ et al. (2009) then showed the coexistence of different wave packets, which separate or not, in these parameter ranges.

The previous studies have considered a plane Poiseuille flow and two-dimensional perturbations. Thanks to a Squire transformation (Jung et al. 1996; Hu et al. 2007), the thresholds associated with more general three-dimensional perturbations can also be deduced. But further studies were needed for real three-dimensional Poiseuille-Rayleigh-Bénard (PRB) flows. Most of these are concerned with pure fluids. In such three-dimensional PRB flows, it has been shown (Gage \& Reid 1968; Luijkx, Platten \& Legros 1981; Platten \& Legros 1984; Nicolas, Luijkx \& Platten 2000) that there exist two main unstable modes which correspond to thermoconvective transverse roll and longitudinal roll patterns. In the case of ducts of infinite lateral extension, the longitudinal rolls appear first since the critical Rayleigh number for these longitudinal rolls, which is independent of the Reynolds number and Prandtl number, is always smaller than the critical Rayleigh number for the transverse rolls, which in contrast increases with the increase of both the Reynolds and Prandtl numbers. For finite rectangular ducts, the lateral confinement tends to stabilize both of these modes, but the threshold (i.e. the critical Rayleigh number) of the longitudinal rolls increases much faster with the confinement than the threshold of the transverse rolls. The consequence is that, for finite rectangular ducts, the transverse rolls appear first at small Reynolds number while the longitudinal rolls appear first at large Reynolds number. Concerning the convective and absolute instability studies, Müller, Lücke \& Kamps (1992) and Müller, Tveitereid \& Trainoff (1993) have first determined the transition curve between the convective and absolute instability zones (AI/CI boundary curve) for the transverse rolls by using a weakly nonlinear theory based on a Ginzburg-Landau equation. Ouazzani, Platten \& Mojtabi (1990) and Ouazzani et al. (1995), experimentally, and Nicolas, Mojtabi \& Platten (1997), numerically, have shown that the transition between the basic flow and the transverse rolls exactly corresponds to the AI/CI boundary curve, provided that the flow is not continuously perturbated at the inlet. Furthermore, for infinite extent system, by evaluating the longtime behaviour of the Green function in the horizontal plane, Carrière \& Monkewitz (1999) theoretically revealed that the mode reaching zero group velocity at the convective-absolute transition always corresponds to transverse rolls, while the system remains convectively unstable with respect to pure streamwise (longitudinal) rolls for all non-zero Reynolds numbers.

In addition to the determination of the linear instability or absolute instability thresholds, it is also important to predict the spatiotemporal patterns that appear in PRB flows, because it is well known that these convection patterns play an important role in CVD reactors where they greatly influence the uniformity of the deposited film thickness. On a linear basis, it is shown that there must exist a cross-over point between the stability boundary curves of both the longitudinal rolls and the transverse rolls in the $R e-R a$ plane, and near the cross-over point, the two modes can set in simultaneously (for an infinite extent system, the cross-over point occurs at $R e=0$ ). In the neighbourhood of the cross-over point, however, the linear stability theory loses its ability to predict convection patterns. This is because a nonlinear interaction between the two coexisting modes plays a significant role in pattern selection. To clarify that, Kato \& Fujimura (2000) derived the amplitude equations for the interaction between 
the longitudinal and the transverse rolls on the basis of a weakly nonlinear theory. The coefficients in the equations are determined numerically for a wide range of parameters. The longitudinal rolls are found to bifurcate supercritically, while the transverse rolls may bifurcate subcritically or supercritically, depending on the Prandtl number, the aspect ratio of the channel, and the boundary conditions on the sidewalls. The different stable convection patterns can then be classified in the parameter space. Note that a mixed mode pattern, which is a mixture of the longitudinal and transverse rolls, is found to be stable for some sets of parameters. These theoretical results greatly supplement those of the linear stability analysis.

So far, it has been reviewed that most studies on the three-dimensional PRB flows concern pure fluids and that, in contrast, the studies devoted to binary fluids consider two-dimensional situations. With the continuous advances in algorithms for the numerical solution of large non-symmetric real/complex generalized eigenvalue problems alongside with the continuous computing hardware improvements, the instability of flows developing in two inhomogeneous and one homogeneous spatial directions can now be analysed (biglobal instability analysis). These biglobal or even triglobal instability analyses have become more and more popular during these recent years for all kinds of flow problems. Such analyses, devoted to different applications, are reviewed by Theofilis $(2003,2011)$. In this paper, the linear biglobal stability analysis is applied to the Rayleigh-Bénard problem for binary fluids with Soret effect, first in cases without throughflow and then in real PRB situations. The objective is to see the influence of both the throughflow and the confinement on the Rayleigh-Bénard instabilities in binary fluids with negative separation factors. The results can also be compared with those obtained for PRB flows in pure fluids. The formulation of the problem is given in $\S 2$. The biglobal instability analysis is then presented in $\S 3$ together with some validations. The results are finally given in the two next sections, first in the case without throughflow and then in the more general PRB situation. Both transverse roll and longitudinal roll structures have been considered. Without throughflow, a given transverse roll structure remains the dominant mode, whereas with throughflow different dominant modes can be found depending on the confinement and the Reynolds number.

\section{Formulation}

\subsection{Governing equations for PRB duct flows}

We consider a rectangular duct with height $H$ (along $z$ ) and width $L$ (along $y$ ) which is filled with a binary mixture and is heated from below (a temperature $T_{2}$ is applied at the upper wall and $T_{1}>T_{2}$ is applied at the bottom; see the schematic representation in figure 1). A steady laminar flow may be generated inside the duct by imposing a constant pressure gradient along the homogeneous spatial $x$-direction. Owing to the influence of the gravitational effect, the binary mixture may become unstable under the influence of vertical temperature and concentration gradients. To take this into account, the density variations are considered, but, according to the Boussinesq approximation, they are restricted to the buoyancy term and are expressed as a linear law,

$$
\rho=\rho_{0}\left[1-\beta_{T}\left(T-T_{0}\right)-\beta_{C}\left(C-C_{0}\right)\right],
$$

where $\beta_{T}$ and $\beta_{C}$ are the thermal and solutal expansion coefficients; $\rho_{0}, T_{0}$ and $C_{0}$ are reference values for density, temperature and concentration, respectively, which are taken as the mean initial values of the respective fields. 


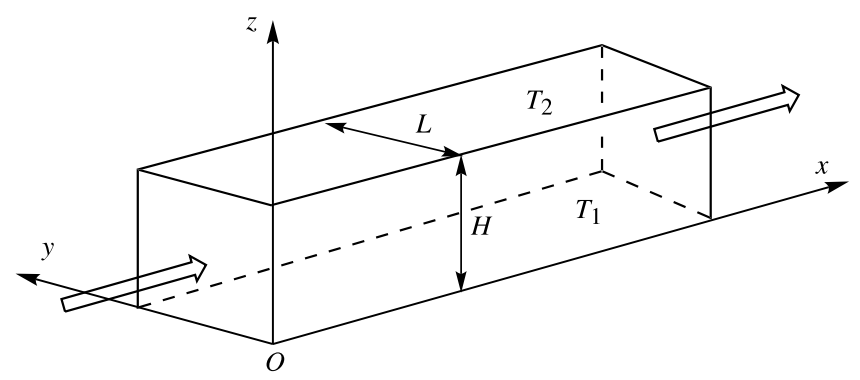

FIGURE 1. Schematic representation of the PRB model.

The Soret effect, which arises as the contribution of the temperature gradient to the mass flux, is considered here, whereas the Dufour effect, which arises as the contribution of the concentration gradient to the heat flux, is neglected. This assumption is valid for liquid mixtures. The mass flux $J_{C}$ and the heat flux $J_{T}$ are then

$$
\begin{gathered}
J_{C}=-\rho_{0} D_{C} \nabla C-\rho_{0} D_{S} \nabla T, \\
J_{T}=-D_{T} \nabla T,
\end{gathered}
$$

where $D_{C}, D_{S}$ and $D_{T}$ are the solutal diffusion coefficient, Soret diffusion coefficient and thermal conductivity, respectively. The conductive steady state will then correspond to linear variations along the vertical $z$-direction for both the temperature and the concentration, leading to a concentration difference $\Delta C=-D_{S} \Delta T / D_{C}$ induced by the applied temperature difference $\Delta T=T_{1}-T_{2}$.

The flow in this system is modelled by the Navier-Stokes equations coupled to an energy equation and a concentration equation. In these equations, length, velocity, time and pressure are scaled by $H, \kappa / H, H^{2} / \kappa$ and $\rho_{0} \kappa^{2} / H^{2}$, respectively $(\kappa$ is the thermal diffusivity). The dimensionless temperature $\theta$ and concentration $c$ are defined as $\left(T-T_{0}\right) / \Delta T$ and $\left(C-C_{0}\right) / \Delta C$. Thus, the dimensionless governing equations of the three-dimensional PRB flow are

$$
\begin{gathered}
\boldsymbol{\nabla} \cdot \boldsymbol{v}=0 \\
\frac{\partial \boldsymbol{v}}{\partial t}+(\boldsymbol{v} \cdot \boldsymbol{\nabla}) \boldsymbol{v}=-\nabla p+\operatorname{Pr} \nabla^{2} \boldsymbol{v}+\operatorname{Ra} \operatorname{Pr}(\theta+\psi c) \boldsymbol{e}_{z}, \\
\frac{\partial \theta}{\partial t}+\boldsymbol{v} \cdot \boldsymbol{\nabla} \theta=\nabla^{2} \theta, \\
\frac{\partial c}{\partial t}+\boldsymbol{v} \cdot \nabla c=L e\left(\nabla^{2} c-\nabla^{2} \theta\right),
\end{gathered}
$$

where $\boldsymbol{v}=(u, v, w)$ is the three-dimensional dimensionless velocity vector, $\boldsymbol{e}_{z}$ is the unit vector in the vertical direction, and the operators are defined as $\nabla=\left(\partial_{x}, \partial_{y}, \partial_{z}\right)$ and $\nabla^{2}=\partial_{x}^{2}+\partial_{y}^{2}+\partial_{z}^{2}$. The dimensionless parameters appearing in the governing system (2.3) are the Prandtl number, $P r=v / \kappa$, the Rayleigh number, $R a=\beta_{T} g H^{3} \Delta T / \kappa \nu$, the separation factor, $\psi=-\beta_{C} D_{S} / \beta_{T} D_{C}$ and the Lewis number, $L e=D_{C} / \kappa$. Here, $v$ is the kinematic viscosity. Ethanol-water mixtures are very convenient for studying Soret-driven flows, because the separation factor can be varied over a wide range by changing the average ethanol concentration. For typical experimental conditions, $5<P r<11$ and $L e \approx 0.01$, so that $P r=10$ and $L e=0.01$ are usually used to represent the values of the Prandtl number and Lewis number for ethanol-water mixtures. These are the values we will choose in our study. The separation factor $\psi$ 
will be chosen as negative, a case where symmetric left and right TWs are found for $R e=0$, and its value will be fixed to $\psi=-0.01$.

The section of the rectangular duct is now defined in dimensionless units by $\Omega=y \in[0, A] \times z \in[0,1]$, where $A=L / H$ is the aspect ratio of the duct. The boundary conditions associated with $(2.3)$ are then

$$
\begin{array}{rcl}
\text { no-slip conditions: } & u=v=w=0 & \text { at } z=0,1 \text { and } y=0, A, \\
\text { thermal conditions: } & \theta=0.5 & \text { at } z=0, \\
& \theta=-0.5 & \text { at } z=1, \\
& \partial_{y} \theta=0 & \text { at } y=0, A, \\
\text { mass impermeability: } & \partial_{z} \theta-\partial_{z} c=0 & \text { at } z=0,1, \\
& \partial_{y} \theta-\partial_{y} c=0 & \text { at } y=0, A,
\end{array}
$$

where $\partial_{y}$ and $\partial_{z}$ denote $\partial / \partial_{y}$ and $\partial / \partial_{z}$, respectively. The non-dimensional basic steady state can easily be obtained by setting the linearly distributed temperature and concentration field along the bounded $z$-direction

$$
\begin{aligned}
& \bar{\theta}(z)=0.5-z, \\
& \bar{c}(z)=0.5-z,
\end{aligned}
$$

and by imposing a constant pressure gradient in the unbounded $x$-direction with a linear profile of pressure gradient in the gravitational $z$-direction,

$$
\nabla \bar{p}=-12 \operatorname{RePr}^{2} \boldsymbol{e}_{x}+\operatorname{Ra} \operatorname{Pr}(1+\psi)(0.5-z) \boldsymbol{e}_{z},
$$

which drives a steady laminar flow, i.e. the Poiseuille flow. Thus, the basic streamwise velocity is solved by the Poisson equation

$$
\nabla_{2 d}^{2} \bar{u}=\frac{1}{\operatorname{Pr}} \partial_{x} \bar{p}=-12 \operatorname{Re} \operatorname{Pr},
$$

where $\nabla_{2 d}^{2}=\partial_{y}^{2}+\partial_{z}^{2}$. The boundary conditions for the basic velocity field are

$$
\bar{u}(y, z=0)=\bar{u}(y, z=1)=\bar{u}(y=0, z)=\bar{u}(y=A, z)=0 .
$$

The above Poisson problem may be solved in series form (Rosenhead 1963), which gives

$$
\begin{aligned}
\bar{u}(y, z)= & \operatorname{Re} \operatorname{Pr}[6 z(1-z) \\
& \left.+\frac{48}{\pi^{3}} \sum_{n=0}^{\infty} \frac{\cosh [(2 n+1) \pi(y-A / 2)] \cos [(2 n+1) \pi(z-1 / 2)]}{(-1)^{n+1}(2 n+1)^{3} \cosh [(2 n+1) \pi A / 2]}\right] .
\end{aligned}
$$

The plane Poiseuille flow result, $\bar{u}(z)=6 \operatorname{Re} \operatorname{Pr}(1-z) z$, is recovered from this expression in the limit $A \rightarrow \infty$. (In fact, this is true for the whole domain $\Omega$ except for the two regions very close to the boundaries $y=0$ and $y=A$.) Here, $R e=U_{0} H / v$ is the Reynolds number and $U_{0}$ is the dimensional mean velocity obtained by integration over the channel height for the plane situation.

\subsection{Symmetries of the problem}

The duct is considered as infinite in the $x$-direction and the basic flow does not depend on $x$. The problem is thus invariant under translations $T_{x_{0}}$ by length $x_{0}$ along 
the $x$-axis. The group generated by the translations is the group $E(1)$. The problem admits two supplementary symmetries: a reflection symmetry $S_{h}$ with respect to the horizontal mid-plane (at $z=0.5$ ) (up-down symmetry) and a reflection symmetry $S_{l}$ with respect to the longitudinal vertical mid-plane (the plane along the $x$-direction, at $y=A / 2$, denoted as the $V_{l}$ plane) (left-right symmetry). The combination of these two reflection symmetries gives a symmetry of rotation of $\pi$ about the longitudinal mid-axis (axis parallel to the $x$ axis at $z=0.5, y=A / 2$ ), which we denote by $R_{x}$. Here $S_{h}, S_{l}$ and $R_{x}$ are defined, respectively, as

$$
\begin{aligned}
& S_{h}:(x, y, z, t) \rightarrow(x, y, 1-z, t), \quad(u, v, w, \theta, c) \rightarrow(u, v,-w,-\theta,-c), \\
& S_{l}:(x, y, z, t) \rightarrow(x, A-y, z, t), \quad(u, v, w, \theta, c) \rightarrow(u,-v, w, \theta, c), \\
& R_{x}: \quad(x, y, z, t) \rightarrow(x, A-y, 1-z, t), \quad(u, v, w, \theta, c) \rightarrow(u,-v,-w,-\theta,-c) .
\end{aligned}
$$

Each symmetry $S_{h}, S_{l}$ and $R_{x}$ defines a $Z_{2}$ group. The symmetry group of our problem, generated by $T_{x_{0}}, S_{h}$ and $S_{l}$, is isomorphic to $E(1) \times Z_{2} \times Z_{2}$. All of these symmetries may be broken at the instability thresholds where bifurcations to new flow states will occur.

\subsection{Linear stability equations for PRB duct flows}

The disturbed three-dimensional PRB flow with Soret effect can be decomposed as $u=\bar{u}+u^{\prime}, v=v^{\prime}, w=w^{\prime}, p=\bar{p}+p^{\prime}, \theta=\bar{\theta}+\theta^{\prime}$ and $c=\bar{c}+c^{\prime}$, where the primes refer to small perturbation quantities. After substituting these variables into the governing equations (2.3) and neglecting the terms which are quadratic with respect to the perturbations, we can obtain the linearized perturbation equations,

$$
\begin{gathered}
\frac{\partial u^{\prime}}{\partial x}+\frac{\partial v^{\prime}}{\partial y}+\frac{\partial w^{\prime}}{\partial z}=0, \\
\frac{\partial u^{\prime}}{\partial t}+\bar{u} \frac{\partial u^{\prime}}{\partial x}+\frac{\mathrm{d} \bar{u}}{\mathrm{~d} y} v^{\prime}+\frac{\mathrm{d} \bar{u}}{\mathrm{~d} z} w^{\prime}=-\frac{\partial p^{\prime}}{\partial x}+\operatorname{Pr} \nabla^{2} u^{\prime}, \\
\frac{\partial v^{\prime}}{\partial t}+\bar{u} \frac{\partial v^{\prime}}{\partial x}=-\frac{\partial p^{\prime}}{\partial y}+\operatorname{Pr} \nabla^{2} v^{\prime}, \\
\frac{\partial w^{\prime}}{\partial t}+\bar{u} \frac{\partial w^{\prime}}{\partial x}=-\frac{\partial p^{\prime}}{\partial z}+\operatorname{Pr} \nabla^{2} w^{\prime}+\operatorname{Ra} \operatorname{Pr}\left(\theta^{\prime}+\psi c^{\prime}\right), \\
\frac{\partial \theta^{\prime}}{\partial t}+\bar{u} \frac{\partial \theta^{\prime}}{\partial x}+\frac{\mathrm{d} \bar{\theta}}{\mathrm{d} z} w^{\prime}=\nabla^{2} \theta^{\prime}, \\
\frac{\partial c^{\prime}}{\partial t}+\bar{u} \frac{\partial c^{\prime}}{\partial x}+\frac{\mathrm{d} \bar{c}}{\mathrm{~d} z} w^{\prime}=\operatorname{Le}\left(\nabla^{2} c^{\prime}-\nabla^{2} \theta^{\prime}\right) .
\end{gathered}
$$

The corresponding boundary conditions are

$$
\begin{array}{rcl}
\text { no-slip conditions: } & u^{\prime}=v^{\prime}=w^{\prime}=0 & \text { at } z=0,1 \text { and } y=0, A, \\
\text { thermal conditions: } & \theta^{\prime}=0 & \text { at } z=0,1, \\
& \partial_{y} \theta^{\prime}=0 & \text { at } y=0, A, \\
\text { mass impermeability: } & \partial_{z} \theta^{\prime}-\partial_{z} c^{\prime}=0 & \text { at } z=0,1, \\
& \partial_{y} \theta^{\prime}-\partial_{y} c^{\prime}=0 & \text { at } y=0, A .
\end{array}
$$

The perturbation quantities can further be expanded as normal modes,

$$
\left(u^{\prime}, v^{\prime}, w^{\prime}, p^{\prime}, \theta^{\prime}, c^{\prime}\right)=[\hat{u}(y, z), \hat{v}(y, z), \hat{w}(y, z), \hat{p}(y, z), \hat{\theta}(y, z), \hat{c}(y, z)] \exp [\mathrm{i}(k x-\omega t)],
$$


where $k$ is a real wavenumber and $\omega$ a complex frequency. Substituting these expressions (2.16) in the governing system, we obtain the linear stability equations expressed as

$$
\begin{gathered}
\mathrm{i} k \hat{u}+D_{y} \hat{v}+D_{z} \hat{w}=0, \\
-\mathrm{i} \omega \hat{u}+\mathrm{i} k \bar{u} \hat{u}+\left(D_{y} \bar{u}\right) \hat{v}+\left(D_{z} \bar{u}\right) \hat{w}=-\mathrm{i} k \hat{p}+\operatorname{Pr}\left(D^{2}-k^{2}\right) \hat{u}, \\
-\mathrm{i} \omega \hat{v}+\mathrm{i} k \bar{u} \hat{v}=-D_{y} \hat{p}+\operatorname{Pr}\left(D^{2}-k^{2}\right) \hat{v} \\
-\mathrm{i} \omega \hat{w}+\mathrm{i} k \bar{u} \hat{w}=-D_{z} \hat{p}+\operatorname{Pr}\left(D^{2}-k^{2}\right) \hat{w}+\operatorname{Ra} \operatorname{Pr}(\hat{\theta}+\psi \hat{c}), \\
-\mathrm{i} \omega \hat{\theta}+\mathrm{i} k \bar{u} \hat{\theta}+\left(D_{z} \bar{\theta}\right) \hat{w}=\left(D^{2}-k^{2}\right) \hat{\theta} \\
-\mathrm{i} \omega \hat{\eta}+\mathrm{i} k \bar{u} \hat{\eta}=\left(D^{2}-k^{2}\right) \hat{\theta}+L e\left(D^{2}-k^{2}\right) \hat{\eta}
\end{gathered}
$$

where $\eta=\theta-c, D_{y}=\partial_{y}, D_{z}=\partial_{z}$ and $D^{2}=\partial_{y}^{2}+\partial_{z}^{2}$. For simplicity, the linear stability equations are abbreviated as

$$
\begin{gathered}
\mathrm{i} k \hat{u}+D_{y} \hat{v}+D_{z} \hat{w}=0, \\
L_{1} \hat{u}-\left(D_{y} \bar{u}\right) \hat{v}-\left(D_{z} \bar{u}\right) \hat{w}-\mathrm{i} k \hat{p}=-\mathrm{i} \omega \hat{u}, \\
L_{1} \hat{v}-D_{y} \hat{p}=-\mathrm{i} \omega \hat{v}, \\
L_{1} \hat{w}-D_{z} \hat{p}+\operatorname{Ra} \operatorname{Pr}[(1+\psi) \hat{\theta}-\psi \hat{\eta}]=-\mathrm{i} \omega \hat{w}, \\
L_{2} \hat{\theta}-\left(D_{z} \bar{\theta}\right) \hat{w}=-\mathrm{i} \omega \hat{\theta}, \\
L_{3} \hat{\eta}+\left(D^{2}-k^{2}\right) \hat{\theta}=-\mathrm{i} \omega \hat{\eta},
\end{gathered}
$$

where $L_{1}=\operatorname{Pr}\left(D^{2}-k^{2}\right)-\mathrm{i} k \bar{u}, L_{2}=\left(D^{2}-k^{2}\right)-\mathrm{i} k \bar{u}$ and $L_{3}=L e\left(D^{2}-k^{2}\right)-\mathrm{i} k \bar{u}$. The corresponding boundary conditions are

$$
\begin{array}{rcl}
\text { no-slip conditions: } & \hat{u}=\hat{v}=\hat{w}=0 & \text { at } z=0,1 \text { and } y=0, A, \\
\text { thermal conditions: } & \hat{\theta}=0 & \text { at } z=0,1, \\
& D_{y} \hat{\theta}=0 & \text { at } y=0, A, \\
\text { mass impermeability: } & D_{z} \hat{\eta}=0 & \text { at } z=0,1, \\
& D_{y} \hat{\eta}=0 & \text { at } y=0, A .
\end{array}
$$

The linear stability (2.17) are two-dimensional partial differential equations, and if there exists a non-trivial solution for the equations, a corresponding dispersion relation

$$
D(k, \omega ; R a, \operatorname{Re}, \psi, \operatorname{Pr}, L e)=0
$$

should be satisfied, and we need to solve a biglobal eigenvalue problem. Because it is impossible to find the explicit analytical dispersion relation if there is no further simplification, the dispersion relation has to be obtained numerically. In this paper, the two-dimensional Chebyshev spectral collocation method (Canuto et al. 2006) is used to discretize the eigenvalue problem and the implicitly restarted Arnoldi method (Lehoucq \& Sorensen 1996) is used to solve the resulting general eigenvalue problem. The algorithms will be described in detail in the next section.

\section{Biglobal instability analysis}

\subsection{Chebyshev spectral collocation method}

First, the physical domain considered here is the domain $\Omega=\{y \in[0, A]\} \times$ $\{z \in[0,1]\}$, which should be transformed into the computational domain 
$\Omega_{c}=\{\xi \in[-1,1]\} \times\{\zeta \in[-1,1]\}$ through a linear transformation relation

$$
\xi=(2 y / A)-1, \quad \zeta=2 z-1 .
$$

Thus, on the computational domain $\Omega_{c}$, we can expand the eigenfunctions in a twodimensional Chebyshev series such as

$$
\hat{c}(y, z)=\sum_{n=0}^{N_{y}} \sum_{m=0}^{N_{z}} a_{n m} T_{n}(\xi) T_{m}(\zeta) .
$$

Here, $T_{n}(\xi)$ and $T_{m}(\zeta)$ are $n$ and $m$ order Chebyshev polynomials and $N_{y}+1$ and $N_{z}+1$ are the numbers of Chebyshev polynomials in the $y$ - and $z$-directions, respectively. The derivatives of the eigenfunctions are obtained by differentiating the expansion above. For example, for the second derivatives, we obtain

$$
\begin{gathered}
\frac{\mathrm{d}^{2}}{\mathrm{~d} y^{2}} \hat{c}(y, z)=\left(\frac{\mathrm{d} \xi}{\mathrm{d} y}\right)^{2} \sum_{n=0}^{N_{y}} \sum_{m=0}^{N_{z}} a_{n m} T_{n}^{\prime \prime}(\xi) T_{m}(\zeta), \\
\frac{\mathrm{d}^{2}}{\mathrm{~d} z^{2}} \hat{c}(y, z)=\left(\frac{\mathrm{d} \zeta}{\mathrm{d} z}\right)^{2} \sum_{n=0}^{N_{y}} \sum_{m=0}^{N_{z}} a_{n m} T_{n}(\xi) T_{m}^{\prime \prime}(\zeta), \\
\frac{\mathrm{d}^{2}}{\mathrm{~d} y \mathrm{~d} z} \hat{c}(y, z)=\left(\frac{\mathrm{d} \xi}{\mathrm{d} y}\right)\left(\frac{\mathrm{d} \zeta}{\mathrm{d} z}\right) \sum_{n=0}^{N_{y}} \sum_{m=0}^{N_{z}} a_{n m} T_{n}^{\prime}(\xi) T_{m}^{\prime}(\zeta),
\end{gathered}
$$

and we do similarly for higher derivatives. The collocation method is used to solve the problem. The collocation points $\xi_{i}$ and $\zeta_{j}$ used to discretize the partial differential stability equations and the linear boundary conditions are the extrema of $T_{N_{y}}$ and $T_{N_{z}}$, given by

$$
\xi_{i}=\cos \frac{i \pi}{N_{y}}, \quad \zeta_{j}=\cos \frac{j \pi}{N_{z}} .
$$

We obtain a generalized eigenvalue problem in a matrix form

$$
\boldsymbol{A x}=\omega \boldsymbol{B} \boldsymbol{x}
$$

(where $\boldsymbol{A}$ and $\boldsymbol{B}$ are non-symmetric complex matrices) which is solved with the wellknown shift-and-invert algorithm. First a shift $\mu$ is chosen close to where we want to find the eigenvalues. If we subtract $\mu \boldsymbol{B} \boldsymbol{x}$ from the eigenvalue equation (3.5), we get

$$
(\boldsymbol{A}-\mu \boldsymbol{B}) \boldsymbol{x}=(\omega-\mu) \boldsymbol{B} \boldsymbol{x} .
$$

If we then multiply with the inverse of this shifted matrix, we get

$$
(\omega-\mu)^{-1} \boldsymbol{x}=(\boldsymbol{A}-\mu \boldsymbol{B})^{-1} \boldsymbol{B} \boldsymbol{x}
$$

which is a standard eigenvalue problem

$$
\boldsymbol{K} \boldsymbol{x}=\theta \boldsymbol{x},
$$

with the matrix $\boldsymbol{K}$ defined by $\boldsymbol{K}=(\boldsymbol{A}-\mu \boldsymbol{B})^{-1} \boldsymbol{B}$ and the eigenvalues $\theta_{j}, j=1, \ldots, n$ given by $\theta_{j}=\left(\omega_{j}-\mu\right)^{-1}$. The largest eigenvalues $\theta_{j}$ of the transformed matrix $\boldsymbol{K}$ now correspond to those eigenvalues $\omega_{j}=\mu+1 / \theta_{j}$ of the original pencil $(\boldsymbol{A}, \boldsymbol{B})$ which are the closest to the shift $\mu$. The shifted and inverted matrix $\boldsymbol{K}$ can be computed by an $L U$ factorization of $(\boldsymbol{A}-\mu \boldsymbol{B})$,

$$
\boldsymbol{P}(\boldsymbol{A}-\mu \boldsymbol{B}) \boldsymbol{Q}=\boldsymbol{L} \boldsymbol{U},
$$


which can be done by using the sparse MATLAB command 'lu' ( $\boldsymbol{P}$ and $\boldsymbol{Q}$ are permutations that make the triangular factors $\boldsymbol{L}$ and $\boldsymbol{U}$ sparse and the factorization numerically stable). In fact, the matrix $\boldsymbol{K}$ can also be computed with the MATLAB command ' $\boldsymbol{K}=$ linsolve $((\boldsymbol{A}-\mu \boldsymbol{B}), \boldsymbol{B})$ ', which uses $L U$ factorization with partial pivoting when $(\boldsymbol{A}-\mu \boldsymbol{B})$ is square and QR factorization with column pivoting otherwise. The standard eigenvalue problem (3.8) is solved with the implicitly restarted Arnoldi method (with MATLAB command 'eigs') to obtain the eigenvalues with largest magnitudes (i.e. 'lm' option for 'eigs').

As mentioned by Theofilis (2003), the major challenge associated with the biglobal instability analysis is the size of this generalized non-symmetric, in general complex, matrix eigenvalue problem. For the problem considered here, there exist six twodimensional eigenfunctions involved in the linear stability equations (2.17). With complex values stored in 16 bytes and for $N_{x}+1=N_{y}+1=N$, the storage requirement for the full dense complex matrices $\boldsymbol{A}$ and $\boldsymbol{B}$ is

$$
2 \times 6^{2} \times N^{4} \times 16 \times 10^{-9} \mathrm{~GB} .
$$

For $N=64$ (value which is commonly used for typical boundary layer flows), this gives about 9.6 GB. This large storage requirement would limit the computation speed of eigenvalues and eigenfunctions. Present computer facilities, however, can satisfy this requirement and are able to solve biglobal instability problems. In our approach, we found two techniques to reduce the storage requirement. The first technique is simply to adopt a sparse matrix storage for our discretized non-symmetric complex matrices $\boldsymbol{A}$ and $\boldsymbol{B}$, which will reduce the storage by half. The second technique is to reduce the number of two-dimensional eigenfunctions from six to five by eliminating the streamwise velocity eigenfunction $\hat{u}$. For that we combine (2.18a) and (2.18b) and get

$$
\mathrm{i} L_{1}\left(D_{y} \hat{v}+D_{z} \hat{w}\right)-k\left(D_{y} \bar{u}\right) \hat{v}-k\left(D_{z} \bar{u}\right) \hat{w}-\mathrm{i} k^{2} \hat{p}=\omega\left(D_{y} \hat{v}+D_{z} \hat{w}\right) .
$$

It is clear that the third derivatives of the eigenfunctions $\hat{v}$ and $\hat{w}$ appear in the new derived equation. This is a small disadvantage because high-order derivatives are sensitive to numerical discretization errors at modest resolution. The corresponding no-slip boundary conditions for $\hat{u}(2.19 a)$ can be changed into

$$
D_{y} \hat{v}+D_{z} \hat{w}=0, \quad \text { at } z=0,1 \text { and } y=0, A .
$$

Finally, the streamwise velocity eigenfunction $\hat{u}$ can be recovered from

$$
\hat{u}=\frac{\mathrm{i}}{k}\left(D_{y} \hat{v}+D_{z} \hat{w}\right)
$$

for $k \neq 0$. It should be noted that the reduced linear stability equations may produce spurious eigenvalues for $k=0$ because equation (3.13) is not valid in this case. In this paper, only TWs with $k \neq 0$ are considered, so that spurious eigenvalues will not affect our analysis.

From the spectra obtained by solving (3.5), we will compute neutral curves (values of $R a$ for which an eigenvalue $\omega$ has its imaginary part $\omega_{i}$ equal to zero whereas all of the other eigenvalues have negative imaginary parts) depending on $k$, but also critical Rayleigh numbers $R a_{c}$ by minimization along $k$. Note that when different eigenmodes are close to critical in a certain domain of the governing parameters, we will often define a neutral curve and a critical Rayleigh number for each of these modes, but it is clear that the true neutral curve and $R a_{c}$ value will be given by the minimum of these different curves and values. 


$\begin{array}{lc}\text { Resolution } & \text { Mode A } \\ 24 \times 24 & \pm 1.4332535922+1.3844131577 \mathrm{i} \\ 28 \times 28 & \pm 1.4332537142+1.3844131715 \mathrm{i} \\ 32 \times 32 & \pm 1.4332537421+1.3844131747 \mathrm{i} \\ 36 \times 36 & \pm 1.4332537504+1.3844131762 \mathrm{i} \\ 40 \times 40 & \pm 1.4332537534+1.3844131767 \mathrm{i} \\ 44 \times 44 & \pm 1.4332537596+1.3844131715 \mathrm{i} \\ 48 \times 48 & \pm 1.4332537553+1.3844131768 \mathrm{i}\end{array}$

Resolution

$24 \times 24$

$28 \times 28$

$32 \times 32$

$36 \times 36$

$40 \times 40$

$44 \times 44$

$48 \times 48$
Mode C

$\pm 2.1119229402+0.8249930660 \mathrm{i}$

$\pm 2.1119248269+0.8249915138 \mathrm{i}$

$\pm 2.1119253499+0.8249911402 \mathrm{i}$

$\pm 2.1119255169+0.8249910206 \mathrm{i}$

$\pm 2.1119255771+0.8249909772 \mathrm{i}$

$\pm 2.1119256066+0.8249909511 \mathrm{i}$

$\pm 2.1119256116+0.8249909515 \mathrm{i}$
Mode B

$$
\begin{aligned}
& \pm 1.7006743356+1.2496657103 \mathrm{i} \\
& \pm 1.7006746271+1.2496655484 \mathrm{i} \\
& \pm 1.7006746820+1.2496655193 \mathrm{i} \\
& \pm 1.7006746989+1.2496655130 \mathrm{i} \\
& \pm 1.7006747054+1.2496655106 \mathrm{i} \\
& \pm 1.7006747069+1.2496655102 \mathrm{i} \\
& \pm 1.7006747094+1.2496655086 \mathrm{i}
\end{aligned}
$$

Mode D

$\pm 2.4525308870+0.0436264770 \mathrm{i}$
$\pm 2.4525304703+0.0436271347 \mathrm{i}$
$\pm 2.4525309750+0.0436268660 \mathrm{i}$
$\pm 2.4525310423+0.0436267111 \mathrm{i}$
$\pm 2.4525310608+0.0436266659 \mathrm{i}$
$\pm 2.4525310707+0.0436266482 \mathrm{i}$
$\pm 2.4525310718+0.0436266406 \mathrm{i}$

TABLE 1. Tests of accuracy for the eigenvalues corresponding to the first four unstable oscillatory modes found for $k=3.14, A=5, R a=2000, \operatorname{Re}=0, \operatorname{Pr}=10, L e=0.01$ and $\psi=-0.01$.

\subsection{Validation for the numerical dispersion relation}

The pure Rayleigh-Bénard situation for binary fluids $(\operatorname{Pr}=10, L e=0.01, \psi=-0.01)$ in a duct without throughflow is first considered by setting $R e=0$. In such a case with negative Soret effect, unstable oscillatory modes, corresponding to symmetry degenerate left and right travelling modes are found. The first four modes found for $A=5, R a=2000$ and $k=3.14$, are labelled as $\mathrm{A}, \mathrm{B}, \mathrm{C}$ and $\mathrm{D}$. The convergence of the four corresponding eigenvalues with the grid size is shown in table 1 . We see that the least stable modes have fastest convergence, and that for the mode $\mathrm{D}$ with the slowest convergence, there is already five valid digits in the eigenvalue for the $32 \times 32$ resolution. Such a $32 \times 32$ grid has been used for most computations in this paper.

The linear temporal growth rates of these four unstable oscillatory modes are plotted as a function of the wavenumber $k$ for the same situation $(A=5, R e=0, R a=2000$, $\operatorname{Pr}=10, L e=0.01$ and $\psi=-0.01)$ in figure 2 . We see that, for this case, the unstable modes $\mathrm{B}$ and $\mathrm{C}$ appear even when the real wavenumber $k \rightarrow 0$, thus giving modes which will be referred to as longitudinal modes, while the unstable modes A and D only occur at moderate wavelengths. For the modes $\mathrm{A}$ and $\mathrm{D}$, there exists only one maximum growth rate which occurs at $k \approx 3.18$ and $k \approx 2.57$, respectively. In contrast for the mode $\mathrm{B}$, there exist two local maximum growth rates which occur at $k=0$ and $k \approx 2.954$, and one local minimum growth rate at $k \approx 1.746$. Finally for the mode $\mathrm{C}$, there exist two local maximum growth rates which occur at $k \approx 2.374$ and $k \approx 2.725$, between which there is a local minimum growth rate at $k \approx 2.62$, and there is another local minimum growth rate at $k=0$.

\section{Results in the case without throughflow $(R e=0)$}

\subsection{Neutral curves}

As shown in the previous section, without throughflow four oscillatory modes, corresponding to symmetry degenerate left and right unstable travelling modes, have 


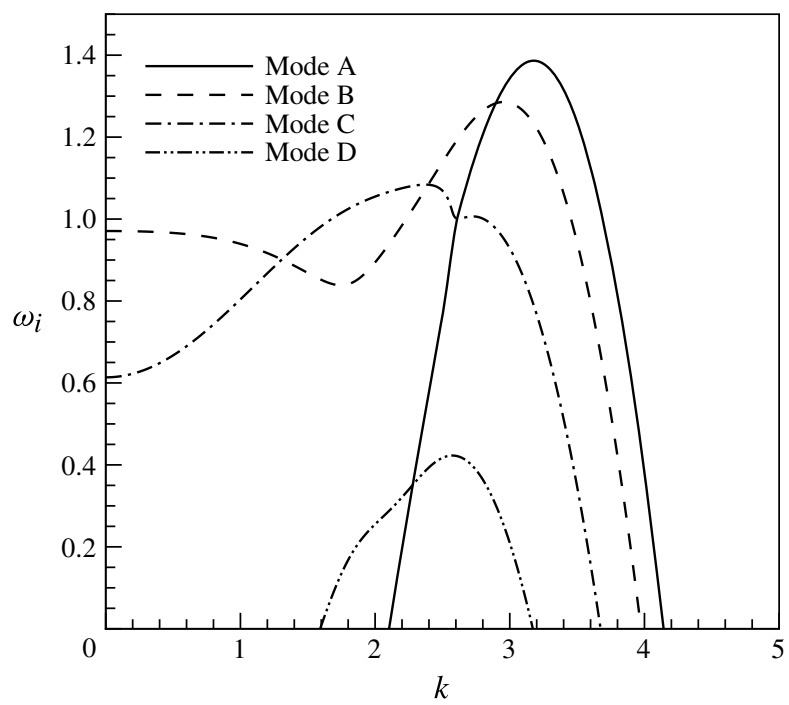

FIGURE 2. Linear temporal growth rates for the first four unstable oscillatory modes as a function of the real wavenumber $k$ when $A=5, R a=2000, \operatorname{Re}=0, \operatorname{Pr}=10, L e=0.01$ and $\psi=-0.01$.

been found for $A=5$ and $R a=2000$. The neutral curves for these four modes, plotted in the parameter plane $k-R a$, are given in figure 3 for different duct aspect ratios $A$. We see that with the increase of the aspect ratio, the four neutral curves for these modes become closer and the parts of these curves corresponding to longitudinal modes (weak $k$ values) eventually disappear from the graph (they could, however, exist for larger $R a$ values). This seems to indicate that the four curves would merge to give a single neutral curve for $A \rightarrow \infty$, i.e. in the two-dimensional limiting case. In fact, for the corresponding two-dimensional Rayleigh-Bénard situation with Soret effect $(\operatorname{Pr}=10, L e=0.01$ and $\psi=-0.01)$, the critical Rayleigh number is $R a_{c}=1743.894$, the critical wavenumber is $k_{c}=3.117$ and the critical angular frequency (denoted as $\omega_{c}$ ) is $\omega_{c}=1.930$. The different curves seem to all evolve towards these critical values as $A$ is increased. The curve of mode $\mathrm{A}$, however, evolves more quickly and the critical values for this mode are already quite close to the two-dimensional critical values for $A=5$.

The spatial structure of these four modes is shown in figure 4 through their temperature distributions at their critical values for a large aspect ratio, $A=10$. These spatial structures are given at a fixed time and are obtained from the eigenvectors at threshold $\left(\omega_{i}=0\right)$. If the discretized eigenvector is denoted as $X=X_{r}+\mathrm{i} X_{i}$, the perturbation for $k$ and $\omega_{r}$ non-equal to zero will be given by $\operatorname{Re}\left(X \mathrm{e}^{\mathrm{i}\left(k x-\omega_{r} t\right)}\right)=X_{r} \cos \left(k x-\omega_{r} t\right)-X_{i} \sin \left(k x-\omega_{r} t\right)$, where Re denotes the real part. For example, for $t=0$, the perturbation would be $X_{r} \cos (k x)-X_{i} \sin (k x)$, i.e. $X_{r}$ for $x=0,-X_{i}$ for $x=\lambda / 4,-X_{r}$ for $x=\lambda / 2$ and $X_{i}$ for $x=3 \lambda / 4$, where $\lambda=2 \pi / k$ is the wavelength. For $t=T / 4, t=T / 2$ and $t=3 T / 4$ (where $T=2 \pi /\left|\omega_{r}\right|$ is the period), we would get perturbations deduced from the perturbation at $t=0$ by a translation of $\lambda / 4$, $\lambda / 2$ and $3 \lambda / 4$, respectively (downstream TW for $\omega_{r}>0$ ).

We see that the four modes shown in figure 4 are clearly different as they have different spatial structures in the cross-section. More precisely, modes A, B, C and $\mathrm{D}$ have two, three, four and five roll structures in the cross-section, respectively. It 

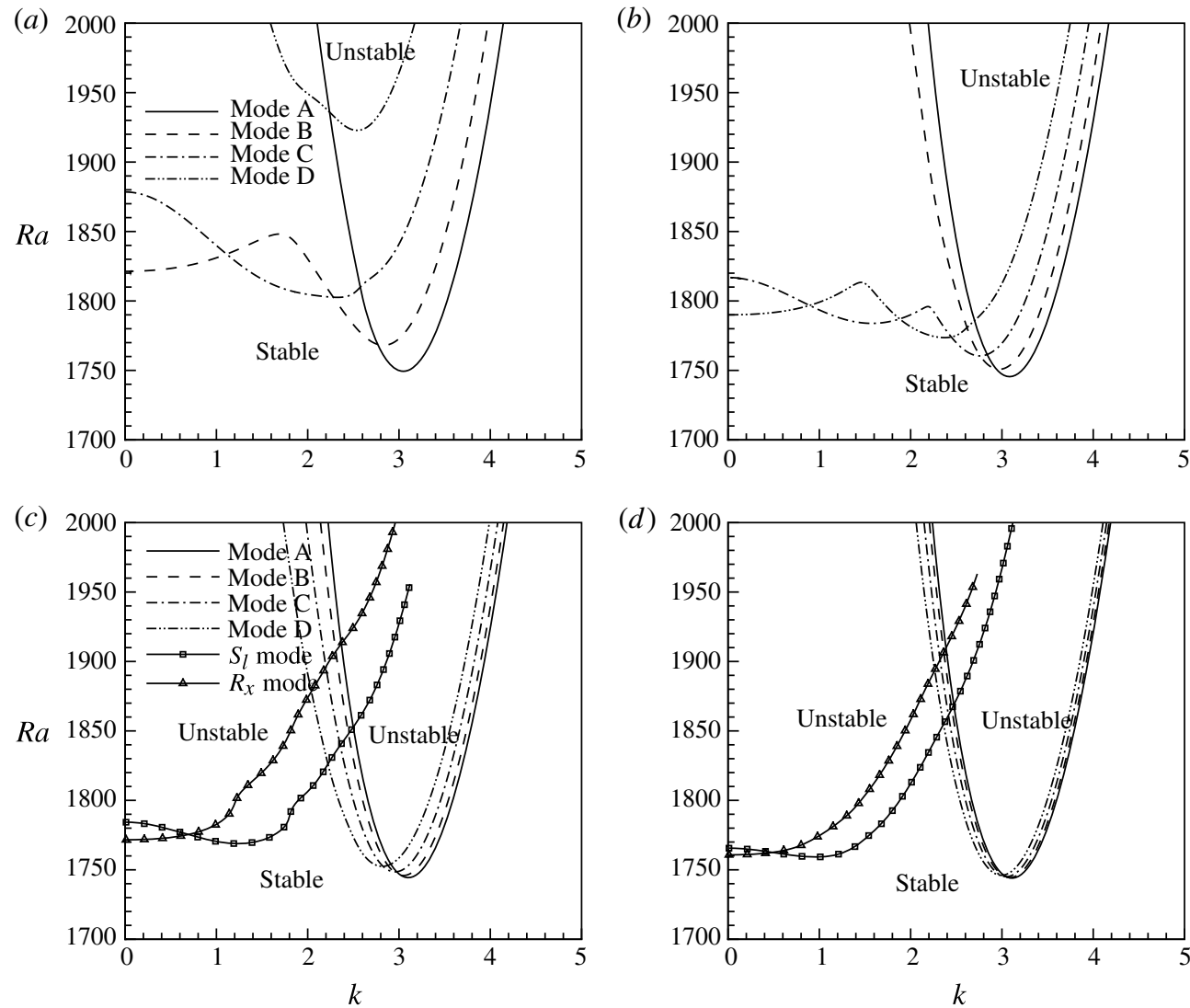

FIGURE 3. Neutral curves for the more unstable oscillatory modes in the parameter plane $k-R a$ for: $(a) A=5 ;(b) A=7 ;(c) A=10$; and $(d) A=15$, when $R e=0, P r=10, L e=0.01$ and $\psi=-0.01$. The first four unstable modes found at finite wavenumber (labelled as A, B, $\mathrm{C}$ and $\mathrm{D}$ modes) are shown, together with the two $S_{l}$ and $R_{x}$ modes which are dominant at small wavenumbers for $A=10$ and 15 .

is interesting to consider the symmetries of these modes. As explained in $\$ 2.2$, the symmetry group of our problem is generated by $T_{x_{0}}, S_{h}$ and $S_{l}$. Each eigenmode with finite wavelength $(k \neq 0)$ breaks the translation invariance $T_{x_{0}}$. All of the eigenmodes obtained here also break the up-down $S_{h}$ symmetry. In contrast, the eigenmodes may break the left-right $S_{l}$ symmetry or not. Among the four modes considered here, modes $\mathrm{A}$ and $\mathrm{C}$ keep the $S_{l}$ symmetry (the temperature perturbation is even under reflection at the plane $y=L / 2$ ), whereas modes B and D break the $S_{l}$ symmetry (the temperature perturbation is odd under reflection at the plane $y=L / 2$ ). The two last modes are in fact symmetric under $R_{x}$. We will then denote all of these modes as $S_{l}$ modes (as A and C modes; these modes will have an even number of rolls) or $R_{x}$ modes (as $\mathrm{B}$ and $\mathrm{D}$ modes; these modes will have an odd number of rolls). We will keep, however, the notation A, B, C, D modes for the four modes which are dominant at finite wavenumber where they correspond to structures in the $y-z$ plane with two, three, four and five rolls, respectively. Finally, note that the critical values given for each mode for $A=10$ in the caption of figure 4 also confirm that the critical values for the mode $\mathrm{A}$ are the closest to those corresponding to the two-dimensional Rayleigh-Bénard situation with Soret effect. 


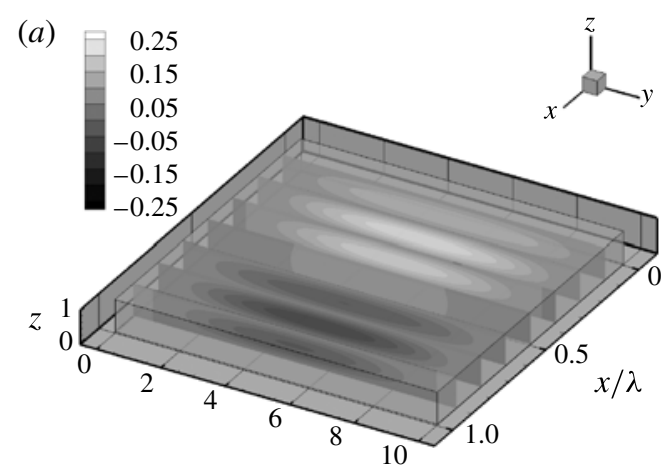

(b)
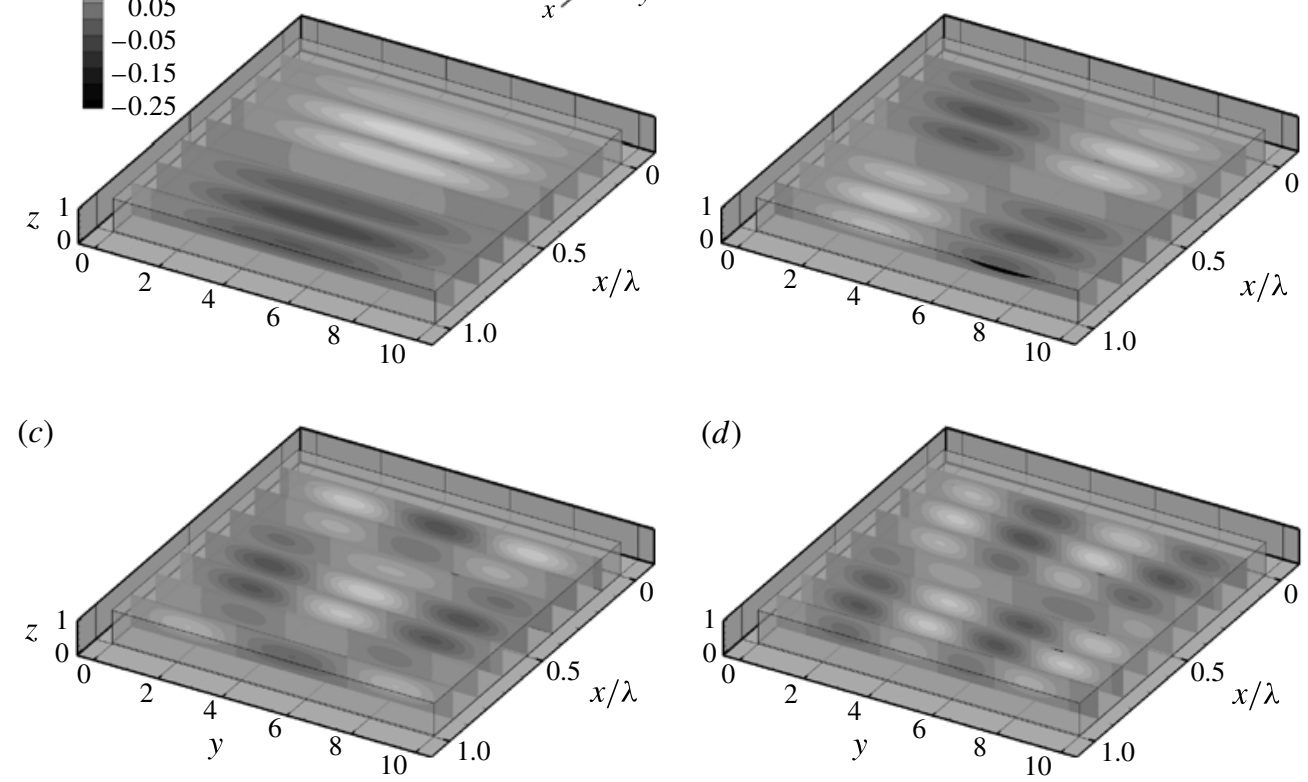

FIGURE 4. Temperature eigenstructure for the first four unstable travelling transverse modes at their critical points for $A=10$ : (a) $R a=1744.38, k=3.100, \omega_{r}=1.932$ (mode A with $S_{l}$ symmetry and two rolls in the cross-section); $(b) R a=1745.90, k=3.048, \omega_{r}=1.935$ (mode B with $R_{x}$ symmetry and three rolls in the cross-section); (c) $R a=1748.55, k=2.957$, $\omega_{r}=1.940$ (mode C with $S_{l}$ symmetry and four rolls in the cross-section); $(d) R a=1752.50$, $k=2.817, \omega_{r}=1.943$ (mode D with $R_{x}$ symmetry and five rolls in the cross-section), when $\operatorname{Re}=0, \operatorname{Pr}=10, L e=0.01$ and $\psi=-0.01$.

The neutral curves shown in figure 3 indicate that both longitudinal modes $(k=0)$ and transverse modes $(k \neq 0)$ issued from the modes $\mathrm{A}, \mathrm{B}, \mathrm{C}$ and $\mathrm{D}$ may exist for moderate aspect ratios such as $A=5$ and $A=7$. It is interesting to analyse the neutral curves which are continuous from moderate to small $k$ values. Those obtained for $A=5$ (figure $3 a$ ) correspond to the $\mathrm{B}$ and $\mathrm{C}$ modes. As shown before, the $\mathrm{B}$ mode is an $R_{x}$ mode with a three roll structure around the minimum of the neutral curve at moderate $k$. This $\mathrm{B}$ mode evolves as $k$ is decreased and changes to a five roll structure, which appears near the maximum of the neutral curve and is well established for $k \approx 0$. The $\mathrm{C}$ mode is an $S_{l}$ mode with a four roll structure which will begin to evolve to a six roll structure near the maximum of the neutral curve at $k=0$. The interesting neutral curves in figure $3(b)$ correspond to the $\mathrm{C}$ and D modes. Similarly, the $\mathrm{C}$ mode has a four roll structure near the main minimum of the neutral curve, which evolves to a six roll structure at the next minimum for smaller $k$ and begin to evolve to a eight roll structure near the maximum at $k=0$. Finally, the $\mathrm{D}$ mode is an $R_{x}$ mode with a five roll structure near the main minimum of the neutral curve and a seven roll structure near the minimum at $k=0$. All this indicates that the number of rolls of the dominant eigenstructure at long wavelength $(k=0)$ changes when $A$ is changed (for example, from five to seven when $A$ is changed from five to seven). We looked for the existence of such long-wavelength eigenstructures for larger values 

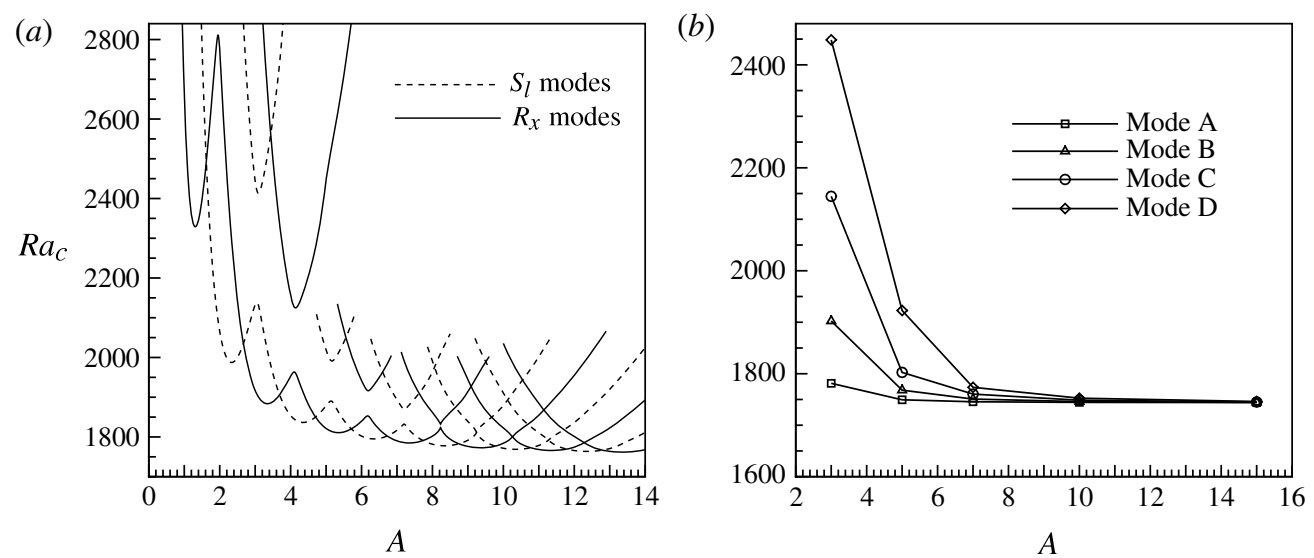

FIGURE 5. Critical curves for the dominant oscillatory modes as a function of the aspect ratio $A$ : $(a)$ longitudinal modes (calculated for $k=0.01$ ); and $(b)$ transverse modes, when $\operatorname{Re}=0, \operatorname{Pr}=10, L e=0.01$ and $\psi=-0.01$.

of $A(A=10$ and $A=15)$ and found new disconnected neutral curves. The neutral curves corresponding to the first two dominant modes in the small $k$ range are plotted in figure 3(c,d) for $A=10$ and $A=15$, respectively. For $A=10$, the dominant mode at small $k$ is a 10 roll $S_{l}$ mode followed by a 9 roll $R_{x}$ mode in transition to 11 rolls, and for $A=15$, the dominant mode at small $k$ is a 14 roll $S_{l}$ mode followed by a 13 roll $R_{x}$ mode in transition to 15 rolls. The coexistence of longitudinal and transverse dominant modes makes it necessary to calculate the critical curves for these two types of modes.

\subsection{Critical curves}

The critical curves for the longitudinal and transverse modes, plotted in the $A-R a_{c}$ plane, are shown in figure 5. Note that the curves for the longitudinal modes are calculated for $k=0.01$.

\subsubsection{Longitudinal modes}

The critical curves for the longitudinal modes (figure $5 a$ ) present oscillatory variations with respect to the aspect ratio $A$. Two main curves determine the minimum values of the critical Rayleigh number $R a_{c}$. One of them is associated with $S_{l}$ modes and the other with $R_{x}$ modes. The regular crossings of the curves as $A$ is increased indicate that the $S_{l}$ and $R_{x}$ modes are alternately the dominant mode. These modes cannot be easily related to the A, B, C and D modes defined previously, as we have shown that the dominant longitudinal modes for $A=5$ and 7 , which both belong to the $R_{x}$ mode critical curve, come from the neutral curves of the $\mathrm{B}$ and $\mathrm{D}$ modes, respectively (see figure 3 ).

Precisions on the structure of the dominant modes can be obtained from the plots of the temperature eigenfunctions (real part) given in figure 6. These eigenfunctions are taken at the true critical threshold (smallest value of $R a_{c}$ ) for different values of $A$. As already mentioned, for $A=5$ and 7 these thresholds belong to the $R_{x}$ mode critical curve, whereas for $A=6$ and 8 they belong to the $S_{l}$ mode critical curve. The critical eigenfunctions shown in figure 6 indicate that the number of rolls of the perturbations increases with $A$ : more precisely, we obtain five, six, seven and eight roll structures 

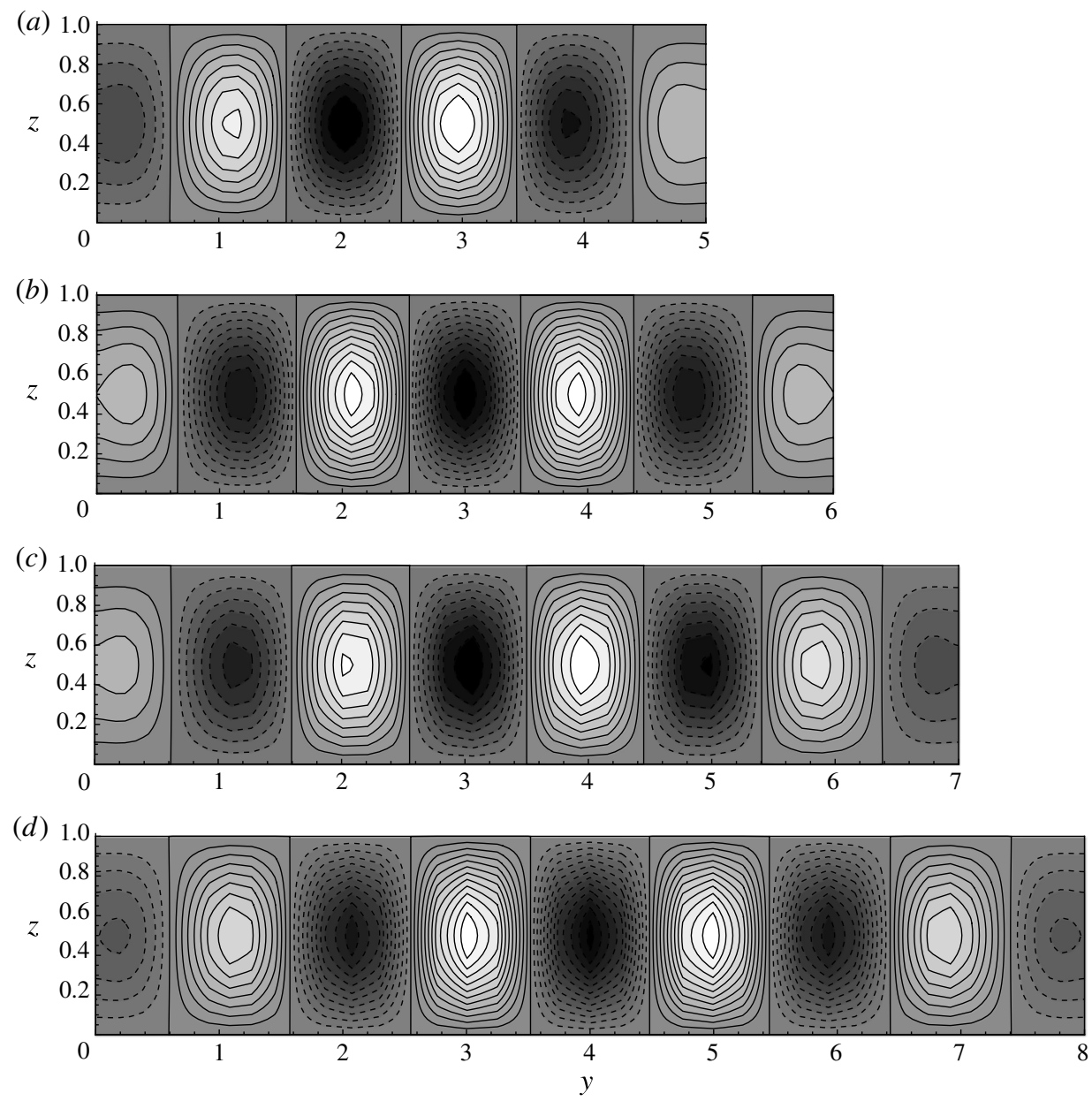

FIGURE 6. Temperature eigenfunction (real part) for the dominant longitudinal modes (calculated for $k=0.01$ ) at threshold for different aspect ratios: (a) $A=5, R a=1821.35$, $\omega_{r}=2.018$ ( $R_{x}$ mode); (b) $A=6, R a=1802.21, \omega_{r}=2.003$ ( $S_{l}$ mode); (c) $A=7$, $R a=1790.01, \omega_{r}=1.992\left(R_{x}\right.$ mode $)$; and $(d) A=8, R a=1781.76, \omega_{r}=1.983\left(S_{l}\right.$ mode $)$, when $\operatorname{Re}=0, \operatorname{Pr}=10, \mathrm{Le}=0.01$ and $\psi=-0.01$.

when $A$ is five, six, seven and eight, respectively. We can also check that the size of the rolls is nearly unchanged when $A$ is increased from five to eight.

In fact, the two main curves in figure $5(a)$ are associated with eigenvectors with different symmetries (they break or not the $S_{l}$ symmetry), so that they can cross at codimension-two bifurcation points. Along each curve, the number of rolls (either odd for the $R_{x}$ mode critical curve or even for the $S_{l}$ mode critical curve) changes by two at the different maxima via the creation or annihilation of two rolls which are initially infinitesimally small. Concerning the upper critical curves, they have a minimum which is just above a maximum of the lower curve with the same symmetry. The gap between such minimum and maximum is due to the phenomenon of avoided crossings between same-symmetry critical curves. This phenomenon, characteristic of convection problems with no-slip lateral boundaries, is expected to occur for pure 
longitudinal modes with $k=0$. The critical curves shown in figure 5(a), however, have been calculated for non-zero $k(k=0.01)$, so that the curves at large $A$ are found to cross each other, which is associated with the disappearance of the expected small gap. Note that for these longitudinal modes with $k \approx 0$, the perturbation for an eigenvector denoted as $X=X_{r}+\mathrm{i} X_{i}$ will be given by $\operatorname{Re}\left(X \mathrm{e}^{-\mathrm{i} \omega_{r} t}\right)=X_{r} \cos \left(\omega_{r} t\right)+X_{i} \sin \left(\omega_{r} t\right)$, so that the perturbation for $\omega_{r}>0$ would be $X_{r}$ for $t=0, X_{i}$ for $t=T / 4,-X_{r}$ for $t=T / 2$ and $-X_{i}$ for $t=3 T / 4$. If we compare the imaginary and real parts of the eigenvectors, we find that they are really similar. For the temperature perturbation, for example, similar positive and negative zones only varying in intensity and slightly in size and position are found. For these longitudinal modes, the oscillations will then correspond to standing waves with counter-rotating rolls periodically changing direction and pulsating in size.

The critical angular frequency $\omega_{c}$ for these oscillatory longitudinal modes is given in table 2. We only give the values associated with the dominant modes for entire values of the aspect ratio $A$. We see that stronger angular frequencies are obtained for small duct widths, but this could be connected to the fact that they are associated with stronger values of $R a_{c}$. The angular frequency decreases as the aspect ratio $A$ is increased stepwise. For $A \rightarrow \infty$, we can expect an asymptotic convergence towards the value $\omega_{c}=1.930$ corresponding to the two-dimensional Rayleigh-Bénard situation with Soret effect. This convergence seems quite slow, with for example a value for $A=10$ which is $\omega_{c}=1.9722$. This slow convergence can be associated with the slow convergence of the thresholds (table 2).

\subsubsection{Transverse modes}

In contrast, the critical curves for the transverse modes (figure $5 b$ ) are monotonous and decrease as $A$ is increased. The critical Rayleigh numbers associated with the first four critical modes are quite different for small aspect ratios as $A=3$. However, when $A$ is increased, they clearly converge to the same value, which is the critical threshold for the two-dimensional Rayleigh-Bénard situation with Soret effect $\left(R a_{c}=1743.894\right)$. Mode A, for which the slowest variation of the threshold with the aspect ratio is found, remains, however, the dominant mode (smallest $R a_{c}$ ) for all aspect ratios. (The good convergence for the mode A when $A$ is increased is also shown in table 3.) These thresholds are also smaller than those found for the longitudinal modes, which indicates that transverse roll structures related to the mode A (i.e. with two rolls in the cross-section) will be the first to appear in these Rayleigh-Bénard flows with Soret effect $(\psi<0)$.

The critical wavenumber $k_{c}$ and angular frequency $\omega_{c}$ for these oscillatory transverse modes are given in table 3 . We see that when $A$ is increased, there is, for all modes, a good convergence towards the values $k_{c}=3.117$ and $\omega_{c}=1.930$ corresponding to the two-dimensional Rayleigh-Bénard situation with Soret effect, the best convergence being, however, obtained for mode A. We find stronger values of the angular frequency $\omega_{c}$ and smaller values of the wavenumber $k_{c}$ for small duct widths. This indicates that, at the onset of the instability, waves with a stronger wave velocity $\omega_{c} / k_{c}$ are obtained for smaller width ducts.

Note finally that the wavelength $\lambda_{l}$ for the dominant longitudinal modes is about two (one more roll when $A$ is increased by one, i.e. $\lambda_{l} / 2=1$ ), which gives a wavenumber $k_{l} \approx \pi$. Similar values are also found for the wavenumber of the dominant transverse modes if $A$ is not too small (see table 3). Such a value of the wavenumber is in fact the value corresponding to the usual pure Rayleigh-Bénard situation $(\psi=0, R e=0)$. 


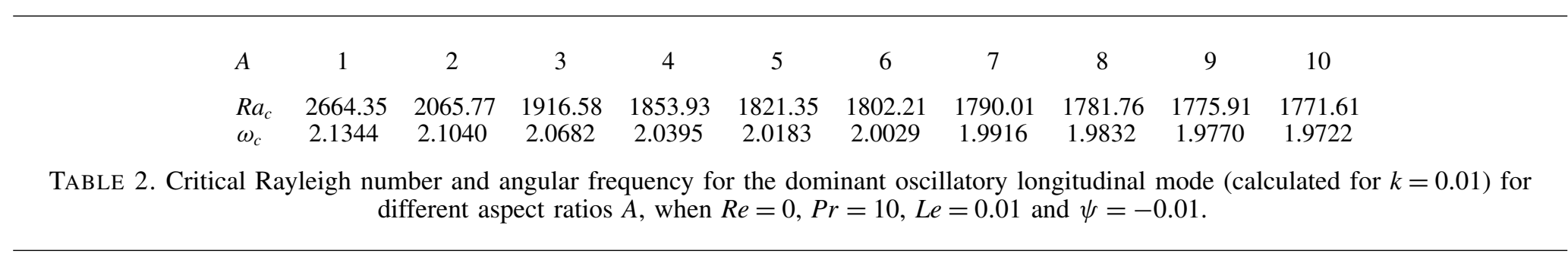
different aspect ratios $A$, when $R e=0, P r=10, L e=0.01$ and $\psi=-0.01$. 
$k_{c}$

$\begin{array}{llllllllll}A & \text { Mode A } & \text { Mode B } & \text { Mode C } & \text { Mode D } & \text { Mode A } & \text { Mode B } & \text { Mode C } & \text { Mode D } & \text { Mode A } \\ 3 & 2.9839 & 2.6994 & 2.6501 & 2.3034 & 1.9818 & 2.1028 & 2.2147 & 2.2678 & 1781.37 \\ 5 & 3.0475 & 2.8309 & 2.3273 & 2.5413 & 1.9399 & 1.9677 & 1.9835 & 2.1633 & 1749.38 \\ 7 & 3.0816 & 2.9702 & 2.7578 & 2.3717 & 1.9340 & 1.9435 & 1.9505 & 1.9514 & 1745.54 \\ 10 & 3.1000 & 3.0480 & 2.9567 & 2.8168 & 1.9315 & 1.9353 & 1.9400 & 1.9429 & 1744.38 \\ 15 & 3.1097 & 3.0873 & 3.0493 & 2.9950 & 1.9307 & 1.9318 & 1.9336 & 1.9358 & 1744.02\end{array}$

TABLE 3. Critical wavenumber and angular frequency for the four dominant oscillatory transverse modes for different aspect ratios $A$, when $R e=0, \operatorname{Pr}=10, L e=0.01$ and $\psi=-0.01$. The critical Rayleigh number for the dominant mode $\mathrm{A}$ is also given.

\subsubsection{Comparison with previous results}

A similar stability study was done by Luijkx \& Platten (1981) in the case of a pure fluid $(\psi=0)$. In that case, the thresholds are not oscillatory thresholds but steady thresholds. Luijkx \& Platten (1981) give the variation of the thresholds with the duct aspect ratio $A$ for both longitudinal and transverse modes. It is interesting to see that they obtain critical curves similar to the curves presented in figure 5, i.e. with a wavy variation for the dominant longitudinal mode (associated with flow structures with an increasing number of rolls) and with a monotonous decrease for the dominant transverse mode, which also has two rolls in the cross-section. In their study, the wavenumber for the dominant transverse mode is also found to increase with the duct aspect ratio in the range $3 \leqslant A \leqslant 15$.

\section{Results in the case with throughflow $(\operatorname{Re} \neq 0)$}

We now investigate the throughflow effect on the critical Rayleigh number for the different dominant modes obtained without throughflow (§4). We first consider this effect for the four dominant transverse modes at $A=5$ and then extend the analysis to the different dominant modes for varying $A$.

\subsection{Effect of Re on the instability of the transverse modes at $A=5$}

The critical curves for the first four unstable oscillatory transverse modes A, B, C and $\mathrm{D}$ as a function of the Reynolds number $R e$ are given for small $|R e|$ and $A=5$ in figure $7(a)$. We recall that for $R e=0$ these oscillatory transverse modes correspond to symmetry degenerate left and right travelling modes. This symmetry is broken by the throughflow, which has a different influence on the thresholds of the left and right travelling modes. In the following, in presence of the throughflow, these left and right travelling modes will rather be presented as upstream and downstream travelling modes, respectively, and they will be often simply denoted as upstream and downstream modes.

The results presented in figure 7(a) have been obtained for modes travelling in the positive $x$-direction and for throughflows either in the positive $(R e>0)$ or negative $(R e<0) x$-direction. If we now refer to the direction of the throughflow, the results obtained for $R e>0(R e<0)$ will be those associated with the effect of the throughflow on the downstream (upstream) modes. For the downstream modes $(R e>0)$, the increase of the throughflow from $R e=0$ induces a continuous increase of their critical Rayleigh numbers. In contrast, for the upstream modes $(R e<0)$, when the throughflow is increased from $R e=0$ (increase of $|R e|$ ) the critical thresholds first 
(a)

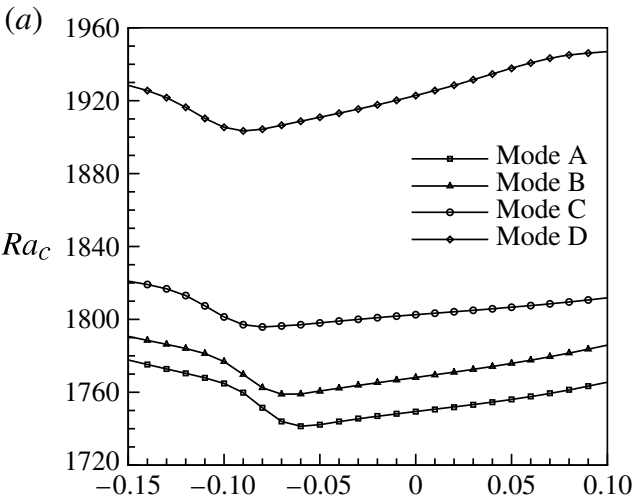

(c)

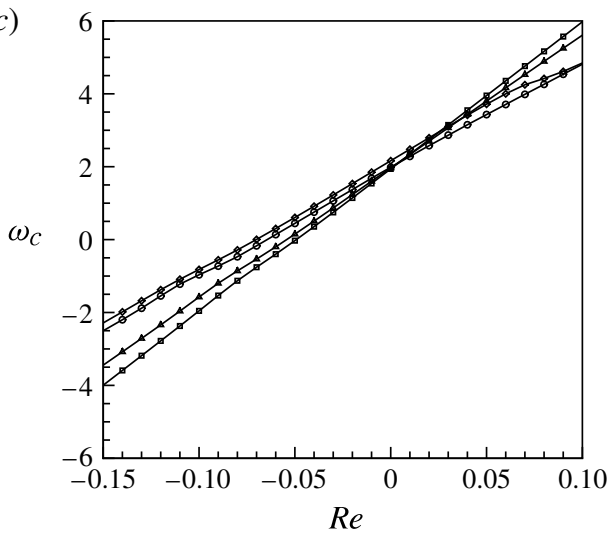

(b)

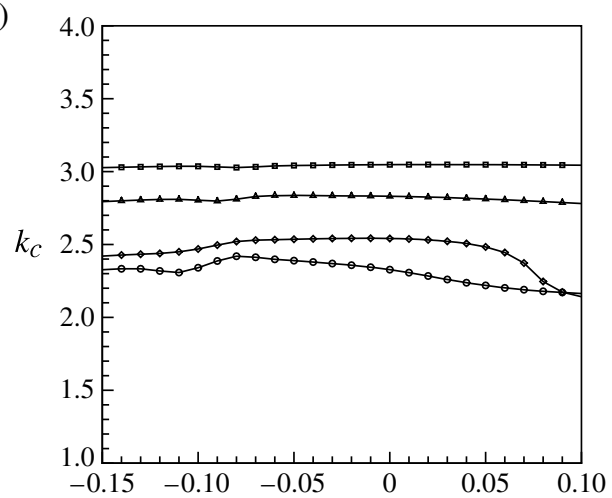

$(d)$

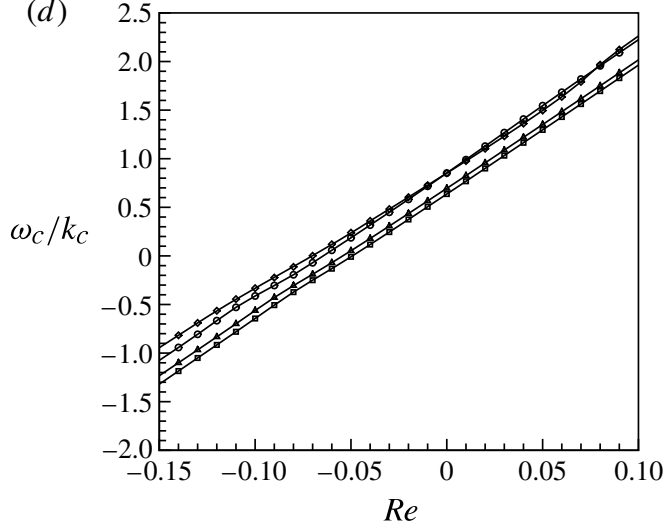

FIGURE 7. Critical Rayleigh number $R a_{c}(a)$, wavenumber $k_{c}(b)$, angular frequency $\omega_{c}$ and phase velocity $\omega_{c} / k_{c}$ for the first four unstable travelling transverse modes as a function of the Reynolds number $\operatorname{Re}$ when $A=5, \operatorname{Pr}=10, L e=0.01$ and $\psi=-0.01$.

decrease, reach a minimum value and then increase more quickly. Note that the critical values associated with the transverse mode A remain the smallest in the $R e$ range studied here, indicating that this mode $\mathrm{A}$ is still the dominant transverse mode.

For these transverse modes, the variations with $R e$ of the critical wavenumber $k_{c}$, angular frequency $\omega_{c}$ and phase velocity $\omega_{c} / k_{c}$ are shown in figure $7(b-d)$. The wavenumber $k_{c}$ (figure $7 b$ ) has only small variations with $R e$. Here $k_{c}$ is even almost constant in the case of the dominant mode $\mathrm{A}$, and the corresponding values are stronger than those obtained for the other modes. The angular frequency $\omega_{c}$ (figure $7 c$ ) increases almost linearly with $R e$, the increase rate being slightly stronger for mode A, followed by mode $\mathrm{B}$, and then modes $\mathrm{C}$ and $\mathrm{D}$. Finally, the phase velocity $\omega_{c} / k_{c}$ (figure $7 d$ ) also increases almost linearly with $R e$, but the increase rate is now similar for the different modes. The phase velocities are slightly smaller for the dominant mode A than for the other modes.

Concerning the longitudinal modes $(k=0)$, they are not influenced by the throughflow, so that their critical thresholds do not depend on $R e$. In fact, as we have calculated these thresholds for $k=0.01$, they might very slightly depend on $R e$. We choose, however, to consider these thresholds as constant and equal to the values already obtained without throughflow in $\$ 4$ (figure $5 a$ ). 


\subsection{Change of dominant modes for varying Re and $A$}

To better see the selective influence of the throughflow on the instabilities, we now compare the critical thresholds obtained for the different dominant modes, i.e. the downstream and upstream transverse modes $\mathrm{A}$ and the dominant longitudinal mode (estimated for $k=0.01$ ). The critical curves for these three modes are plotted as a function of $\operatorname{Re}(R e \geqslant 0)$ for three values of the aspect ratio $A(A=3,5$ and 7$)$ in figure 8 . For $A=3$ (figure $8 a$ ), the critical curve for the upstream transverse mode is below that for the downstream transverse mode and also below the critical value for the longitudinal mode $\left(R a_{c} \approx 1916.58\right.$, slightly outside the $R a_{c}$ range considered in the figure). For $A=3$, the upstream transverse mode is then the dominant mode and its critical curve is the true critical curve within the $R e$ range studied $(R e \leqslant 0.4)$.

For $A=5$ (figure $8 b$ ), the dominant mode in the $R e$ range studied is still the upstream transverse mode. However, the critical curve for the downstream transverse mode is now almost in contact with the critical curve for the upstream transverse mode for Reynolds numbers in the range $0.1 \leqslant R e \leqslant 0.16$. Moreover, the horizontal line corresponding to the critical value for the longitudinal mode $\left(R a_{c} \approx 1821.35\right)$ intersects the critical curve for the downstream transverse mode at $R e=0.258$, indicating that beyond this value, the longitudinal mode is more dangerous than the downstream transverse mode.

Finally, for $A=7$ (figure $8 c$ ), due to different intersections between the critical curves, the dominant mode will depend on the value of $R e$. For small values of $R e$ $(0 \leqslant R e \leqslant 0.09)$, the dominant mode is still the upstream transverse mode. In the range $0.1 \leqslant R e \leqslant 0.19$, because of the crossings between the transverse mode curves, the downstream transverse mode becomes the dominant mode. The upstream transverse mode is again the dominant mode in a small range $0.2 \leqslant R e \leqslant 0.22$. Finally, for the larger values of $R e$ considered in the graph $(0.23 \leqslant R e \leqslant 0.4)$, the longitudinal mode becomes the dominant mode because its critical threshold $R a_{c} \approx 1790.01$ is now below the thresholds for the two transverse modes.

In figure $8(c)$, we also give the critical curves for the dominant modes in the two-dimensional situation $(A \rightarrow \infty)$. We can see that the critical curves for the upstream and downstream transverse modes do not change much for $A \geqslant 7$. There is however a more important crossing between these curves, so that the cross-points which were around $R e=0.092$ and $R e=0.191$ for $A=7$ are around $R e=0.085$ and $R e=0.239$ for $A \rightarrow \infty$. In contrast, there is a clear decrease of the critical value for the longitudinal mode, which changes from $R a_{c} \approx 1790.01$ for $A=7$ to $R a_{c}=1743.894$ for $A \rightarrow \infty$. As a consequence, the four critical $R e$ zones with different unstable modes found for $A=7$ in the range $0 \leqslant R e \leqslant 0.4$ will quickly evolve to three zones for larger values of $A$ : a zone at small $R e$ with the upstream transverse mode, a second zone with the downstream transverse mode, and a final zone with the longitudinal mode. The next and final step for larger values of $A$ will be to have only two critical $R e$ zones, a small zone at small $R e$ with the upstream transverse mode, and a large zone with the longitudinal mode.

Note that in the limiting two-dimensional case (a case corresponding to the dashed curves in figure 8(c) and showing two different critical Re zones), Hu et al. (2007) used a Squire transformation to show that the really expected outcome would be to have a first zone corresponding to $0 \leqslant R e \leqslant R e_{m}$ with the upstream transverse mode $\left(R e_{m}\right.$ is the Reynolds number corresponding to the minimum of the critical curve for the upstream mode, $R a_{m} ; R e_{m}$ is close above 0.05$)$ and a second zone with oblique waves. These oblique waves would appear for $R e \geqslant R e_{m}$ at a fixed threshold $R a_{m}$, but with an angle $\alpha$ varying as $\cos (\alpha)=R e_{m} / R e$, i.e. from $\alpha=0$ to $\pi / 2$ as $R e$ is increased 

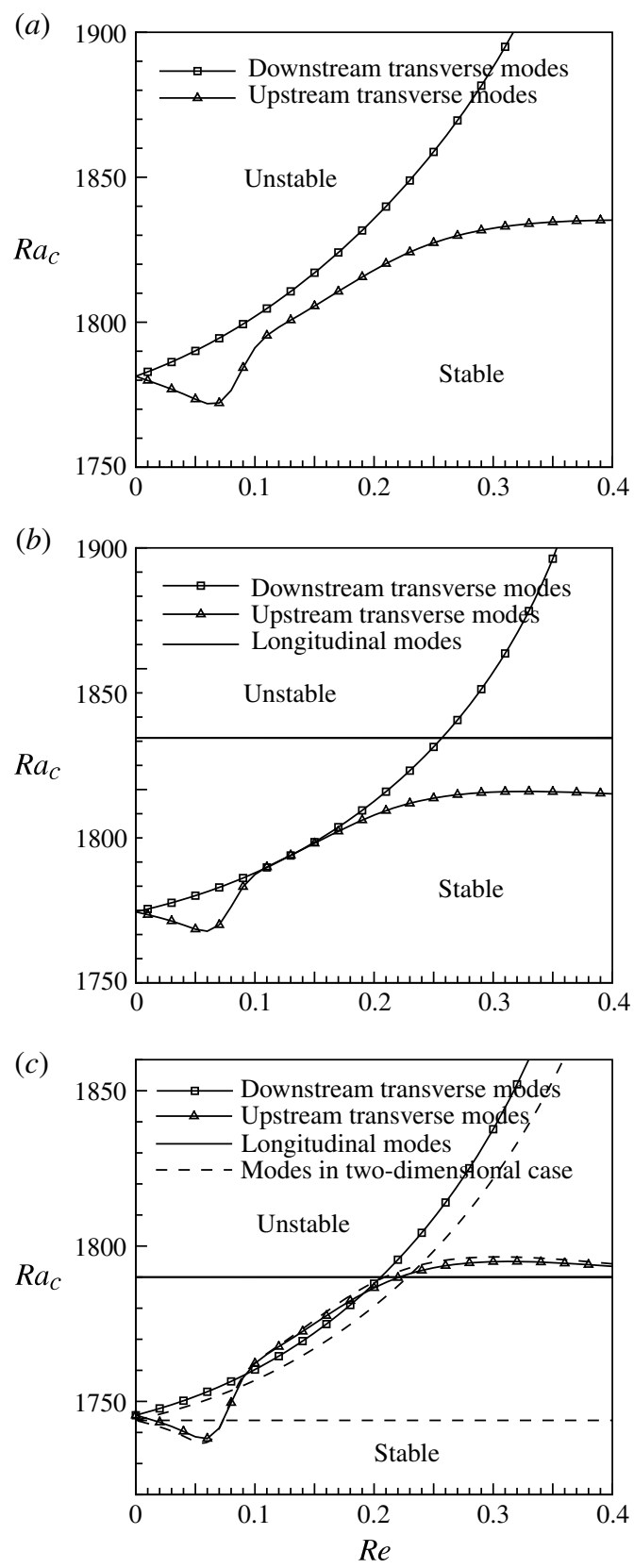

FIGURE 8. Critical curves for the dominant upstream and downstream transverse modes (mode A) and the dominant longitudinal mode $(k=0.01)$ as a function of the Reynolds number $R e$ : (a) $A=3$; (b) $A=5$; and (c) $A=7$, when $P r=10, L e=0.01$ and $\psi=-0.01$. The critical curves for these dominant modes in the two-dimensional situation $(A \rightarrow \infty)$ are also given as dashed lines in $(c)$.

from $R e_{m}$ to $\infty$. These oblique waves would then evolve from the upstream transverse instability (for $R e=R e_{m}$ ) to the longitudinal instability (for $R e \gg R e_{m}$ ). A question 
(a)

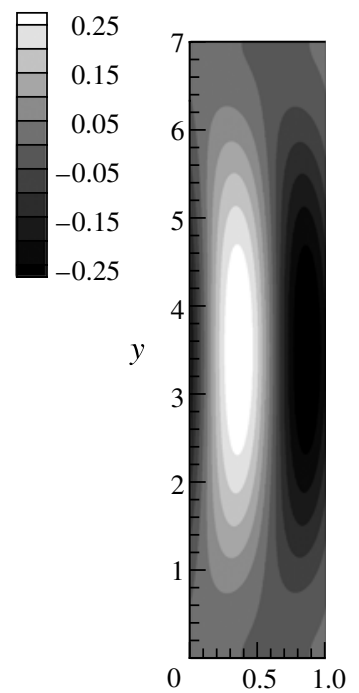

(b)

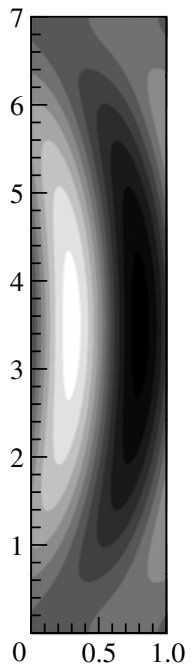

(c)

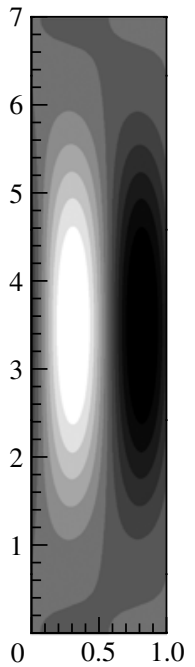

(d)

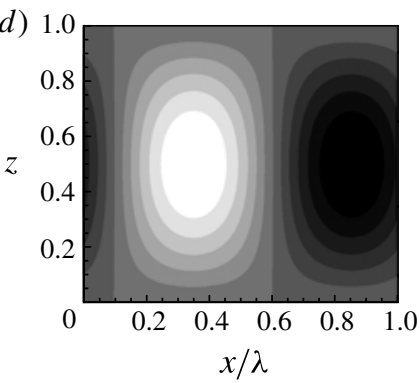

$(e)$

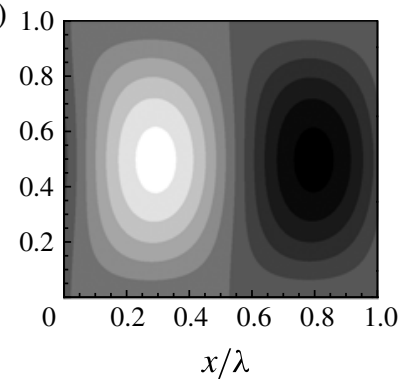

(f)

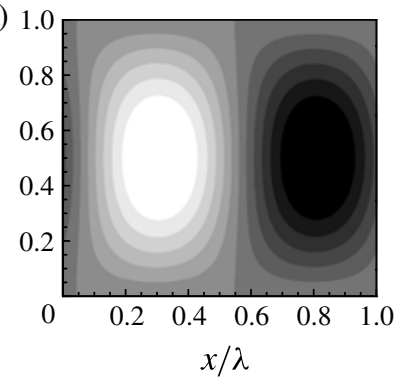

FIGURE 9. Temperature eigenfunction (real part) at threshold for the dominant transverse mode at $\operatorname{Re}=0(a, d)$ and the dominant downstream $(b, e)$ and upstream $(c, f)$ transverse modes at $\operatorname{Re}=0.3$ for $A=7$ when $\operatorname{Pr}=10, L e=0.01$ and $\psi=-0.01$. The isovalues of the temperature are given in the horizontal mid-plane in the upper plots and in the vertical mid-plane $V_{l}$ in the lower plots. For $R e=0$, the downstream mode is shown, although both downstream and upstream modes are equivalent (symmetry degeneracy).

could now be raised: Is it possible to find such oblique waves in ducts with large aspect ratios? We have not yet found clear answers to this question.

Such changes of critical modes as $R e$ is increased also occur in the case without Soret effect (Nicolas et al. 2000). There is, however, only one transition between a downstream transverse mode obtained at small $R e$ and a steady longitudinal mode in this case. The Reynolds number at which this transition occurs is found to increase as the duct aspect ratio increases.

Precisions on the structure of the downstream and upstream transverse modes obtained with throughflow are given in figures 9 and 10 with plots of the temperature and concentration eigenfunctions, respectively. We plot the eigenstructures of the downstream and upstream transverse modes at threshold for $A=7$ and $R e=0.3$ and compare them with those obtained for the dominant transverse mode at $R e=0$ (the downstream mode is shown in the figures, although both downstream and upstream modes are equivalent at $R e=0$ (symmetry degeneracy)). The isovalues 
(a)

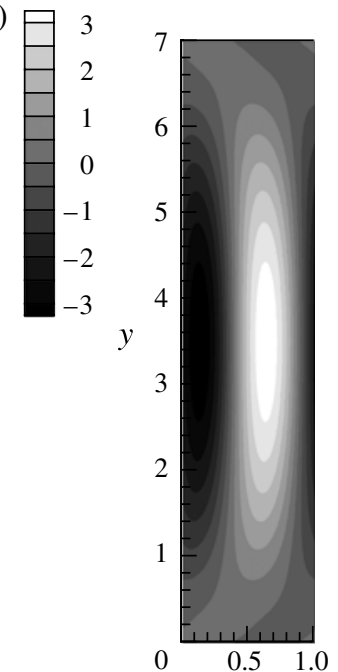

(d)

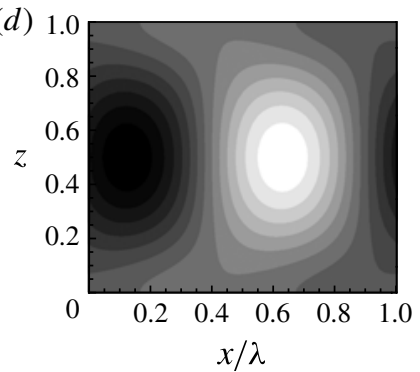

(b)

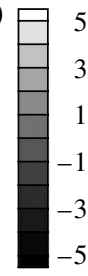

5 3 1

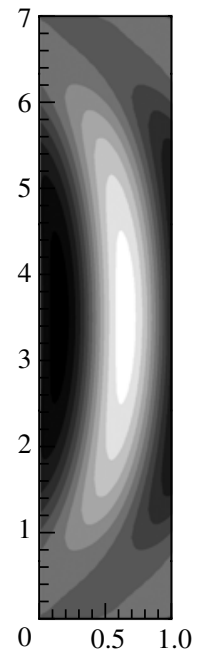

(e)

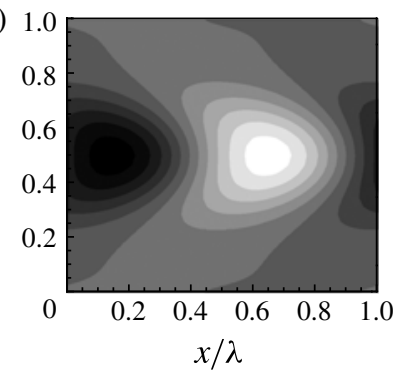

(c)
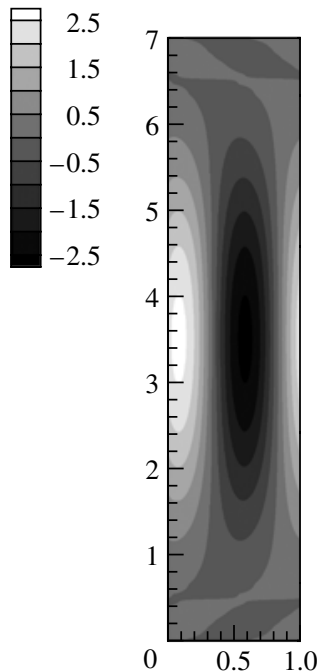

(f)

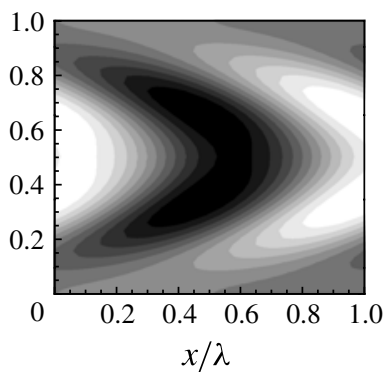

FIGURE 10. Concentration eigenfunction (real part) at threshold for the dominant transverse mode at $R e=0(a, d)$ and the dominant downstream $(b, e)$ and upstream $(c, f)$ transverse modes at $\operatorname{Re}=0.3$ for $A=7$ when $\operatorname{Pr}=10, L e=0.01$ and $\psi=-0.01$. The isovalues of the concentration are given in the horizontal mid-plane in the upper plots and in the vertical mid-plane $V_{l}$ in the lower plots. For $R e=0$, the downstream mode is shown, although both downstream and upstream modes are equivalent (symmetry degeneracy).

of temperature and concentration in the vertical mid-plane $V_{l}$ look similar to those obtained in the two-dimensional case and shown in the paper of Jung et al. (1996). We see the different phase shifts between the concentration and temperature fields for the downstream and upstream modes. The temperature fields in the $V_{l}$ plane for the different modes look roughly similar. In contrast, the concentration fields look different. The effect of the throughflow induces an arrow shape for the concentration field in the $V_{l}$ plane. This effect is less pronounced for the downstream mode, which has concentration variations rather confined in the bulk. The effect is strong for the upstream mode, which has more extended concentration variations in the $V_{l}$ plane. Our results also allow to see the variations of the eigenstructures along the duct width. The temperature and concentration fields appear to be bent along the transverse direction by the throughflow in the case of the downstream transverse mode. Such a bending is not found in the case of the upstream transverse mode and the temperature and concentration fields appear less extended in the transverse direction. 


\section{Conclusions}

In this paper, the three-dimensional Rayleigh-Bénard instabilities in a binary fluid with negative Soret coefficient have been studied by linear biglobal stability analysis in the case of a finite width duct, with and without throughflow. The numerical dispersion relation associated with this stability problem has been obtained with a twodimensional Chebyshev collocation method, and neutral and critical curves have thus been calculated. Using symmetry considerations, the associated critical perturbation modes, which are all oscillatory modes, have been classified as $S_{l}$ modes (those which keep the left-right symmetry) or $R_{x}$ modes (those which keep the symmetry of rotation of $\pi$ about the longitudinal mid-axis).

Without throughflow, four dominant pairs of symmetry degenerate travelling transverse modes with finite wavenumbers $k$ have been found. These pairs of modes have different numbers of rolls in the duct cross-section. With the increase of the duct aspect ratio $A$, the critical Rayleigh numbers for these four pairs of modes decrease monotonously and closely approach the critical value $R a_{c}=1743.894$ obtained in a two-dimensional situation. One of these modes (a $S_{l}$ mode called mode A and with two rolls in the cross-section) is the really dominant transverse mode. This mode A corresponds to the smallest critical values and has the quickest convergence towards the two-dimensional thresholds. Oscillatory longitudinal modes (corresponding to $k \approx 0$ ) have also been found for increasing aspect ratios. The dominant longitudinal modes belong to two wavy critical curves globally decreasing and corresponding to either $S_{l}$ modes with an even number of rolls or $R_{x}$ modes with an odd number of rolls. These two curves interlace with each other so that the true critical thresholds for these longitudinal modes correspond to modes with an increasing number of longitudinal rolls. In this case without throughflow, the smaller thresholds are found for the travelling transverse mode A, which is then expected to be observed.

When a throughflow is applied (three-dimensional PRB flow with Soret effect), the symmetry degeneracy of the pairs of travelling transverse modes is broken, giving distinct upstream and downstream travelling modes, as it was already observed for the two-dimensional PRB flows. The throughflow modifies the thresholds of both the upstream and downstream travelling transverse modes, eventually increasing them. In contrast, it has no effect on the longitudinal mode thresholds. As a consequence, a competition between the different modes can be expected. It is found that for small and moderate aspect ratios $A$, the overall critical Rayleigh number in the small Reynolds number range studied $(0 \leqslant R e \leqslant 0.4)$ is only determined by the upstream transverse mode A. In contrast, for larger aspect ratios different modes are successively dominant as the Reynolds number is increased, involving both upstream and downstream transverse modes A and even the longitudinal mode. Four different $R e$ zones corresponding to different critical modes are found for $A=7$. The number of zones is reduced to three, and then to two when the aspect ratio is further increased. Note that the upstream transverse mode A is always the critical mode for small Reynolds numbers whatever the value of the aspect ratio $A$. And for large values of $A$, the longitudinal mode eventually becomes the critical mode when the Reynolds number is increased. Note finally that the crossings between the different critical curves indicate the existence of $R e$ regions where different transverse and longitudinal modes become unstable simultaneously. From the work of Kato \& Fujimura (2000), it can be expected that a complex nonlinear interaction between the coexisting modes would play a significant role in pattern selection around these $R e$ regions.

This three-dimensional PRB flow situation involving binary fluids with negative Soret coefficients has appeared to be a very rich situation. Depending on the values 
of the governing parameters, principally the duct aspect ratio, the Reynolds number and the Soret coefficient, very different oscillatory perturbations, either longitudinal or transverse, upstream or downstream, are expected to be triggered. It would be now very interesting to perform three-dimensional simulations to confirm all of these results.

\section{Acknowledgements}

We are grateful to the anonymous referees for suggestions that significantly improved the paper. This work is supported by the National Natural Science Foundation of China (NSFC; grants 11172049 and 11072040) and the Science Foundation of CAEP (grants 2011B0201042 and 2012B0201027).

\section{REFERENCES}

BÜCHel, P. \& LÜCKE, M. $2000 a$ Influence of through flow on binary fluid convection. Phys. Rev. E 61, 3793-3810.

BÜCHEL, P. \& LÜCKE, M. $2000 b$ Localized perturbations in binary fluid convection with and without throughflow. Phys. Rev. E 63, 016307.

Canuto, C., Hussaini, M. Y., Quarteroni, A. \& ZAng, T. A. 2006 Spectral Methods: Fundamentals in Single Domains. Springer.

CArrière, Ph. \& Monkewitz, P. A. 1999 Convective versus absolute instability in mixed Rayleigh-Bénard-Poiseuille convection. J. Fluid Mech. 384, 243-262.

Cross, M. C. \& Hohenberg, P. C. 1993 Pattern formation outside of equilibrium. Rev. Mod. Phys. 65, 851-1112.

Gage, K. S. \& REID, W. H. 1968 The stability of thermally stratified plane Poiseuille flow. J. Fluid Mech. 33, 21-32.

Hu, J., Ben Hadid, H. \& Henry, D. 2007 Linear stability analysis of Poiseuille-Rayleigh-Bénard flows in binary fluids with Soret effect. Phys. Fluids 19, 034101.

Hu, J., Yin, X. Y., Ben Hadid, H. \& Henry, D. 2009 Spatiotemporal evolution of Poiseuille-Rayleigh-Bénard flows in binary fluids with Soret effect under initial pulselike disturbances. Phys. Rev. E 80, 026312.

JUNG, CH., LÜCKE, M. \& BÜCHEL, P. 1996 Influence of through-flow on linear pattern formation properties in binary mixture convection. Phys. Rev. E 54, 1510-1529.

JUNG, D. \& LÜCKE, M. 2005 Traveling wave fronts and localized traveling wave convection in binary fluid mixtures. Phys. Rev. E 72, 026307.

Kato, Y. \& FujimurA, K. 2000 Prediction of pattern selection due to an interaction between longitudinal rolls and transverse modes in a flow through a rectangular channel heated from below. Phys. Rev. E 62, 601-611.

LehoucQ, R. B. \& Sorensen, D. C. 1996 Deflation techniques for an implicitly re-started Arnoldi iteration. SIAM J. Matrix Anal. Appl. 17, 789-821.

LuiJkx, J. M. \& Platten, J. K. 1981 On the onset of free convection in a rectangular channel. J. Non-Equilib. Thermodyn. 6, 141-158.

LuiJkx, J. M., Platten, J. K. \& Legros, J. C. 1981 On the existence of thermoconvective rolls, transverse to a superimposed mean Poiseuille flow. Intl J. Heat Mass Transfer 24, 1287-1291.

MÜlleR, H. W., LÜCKE, M. \& KAMPS, M. 1992 Transversal convection patterns in horizontal shear flow. Phys. Rev. A 45, 3714-3726.

Müller, H. W., TVeitereid, M. \& Trainoff, S. 1993 Rayleigh-Bénard problem with imposed weak through-flow: two coupled Ginzburg-Landau equations. Phys. Rev. E 48, 263-272.

Nicolas, X., LuijkX, J. M. \& Platten, J. K. 2000 Linear stability of mixed convection flows in horizontal rectangular channels of finite transversal extension heated from below. Intl J. Heat Mass Transfer 43, 589-610. 
Nicolas, X., Mojtabi, A. \& Platten, J. K. 1997 Two-dimensional numerical analysis of the Poiseuille-Bénard flow in a rectangular channel heated from below. Phys. Fluids 9, 337-348.

Ouazzani, M. T., Platten, J. K. \& Mojtabi, A. 1990 Etude expérimentale de la convection mixte entre deux plans horizontaux à températures différentes - II. Intl J. Heat Mass Transfer 33, 1417-1427.

Ouazzani, M. T., Platten, J. K., Müller, H. W. \& Lücke, M. 1995 Etude de la convection mixte entre deux plans horizontaux à températures différentes - III. Intl J. Heat Mass Transfer 38, 875-886.

Platten, J. K. \& Legros, J. C. 1984 Convection in Liquids. Springer.

Rosenhead, L. 1963 Laminar Boundary Layers. Oxford University Press.

TheofiLIS, V. 2003 Advances in global linear instability analysis of nonparallel and threedimensional flows. Prog. Aerosp. Sci. 39, 249-315.

Theofilis, V. 2011 Global linear instability. Annu. Rev. Fluid Mech. 43, 319-352. 